\title{
SINGULARLY PERTURBED BOUNDARY VALUE PROBLEMS WITH ANGULAR LIMITING SOLUTIONS ${ }^{1}$
}

\author{
BY \\ F. A. HOWES \\ Dedicated to the Memory of Professor Norman Levinson
}

\begin{abstract}
A basic result of Haber and Levinson which describes the behavior of solutions of $\varepsilon y^{\prime \prime}=f\left(t, y, y^{\prime}\right), a<t<b, y(a, \varepsilon), y(b, \varepsilon)$ prescribed, in the presence of a reduced solution with corners is modified to treat related classes of problems. Under various stability assumptions, solutions are shown to remain, for small $\varepsilon>0$, in a $o$ (1)-neighborhood of an angular reduced solution with the possible exception of narrow layers near the boundaries in some cases. Each aspect of the theory developed here is illustrated by several examples.
\end{abstract}

1. Introduction. The well known study of Haber and Levinson [4] investigates the existence and the asymptotic behavior of solutions of the boundary value problem

$$
\begin{gathered}
\varepsilon y^{\prime \prime}=f\left(t, y, y^{\prime}\right), \quad a<t<b, \\
y(a, \varepsilon)=A, \quad y(b, \varepsilon)=B,
\end{gathered}
$$

as the positive parameter $\varepsilon$ tends to zero, when the corresponding reduced problem

$$
\begin{gathered}
f\left(t, u, u^{\prime}\right)=0, \quad a<t<b, \\
u(a)=A, \quad u(b)=B,
\end{gathered}
$$

has an "angular" solution $u=u(t)$. To be precise, the function $u$ is assumed to be of the form $u(t)=u_{L}(t), a \leqslant t \leqslant t_{0}$, and $u(t)=u_{R}(t), t_{0} \leqslant t \leqslant b$, $a<t_{0}<b$, where $u_{L}, u_{R}$ satisfy (1.3) on $\left[a, t_{0}\right]$, $\left[t_{0}, b\right]$, respectively, and $u_{L}(a)=A, u_{R}(b)=B$. In addition, $u_{L}$ and $u_{R}$ are assumed to intersect with unequal slopes at $t=t_{0}$, i.e., $u_{L}\left(t_{0}\right)=u_{R}\left(t_{0}\right)$ and $u_{L}^{\prime}\left(t_{0}\right) \neq u_{R}^{\prime}\left(t_{0}\right)$. Finally if these solutions are stable in the sense that $f_{y^{\prime}}\left[u_{L}(t)\right]=\left(\partial f / \partial y^{\prime}\right)\left(t, u_{L}(t), u_{L}^{\prime}(t)\right)$ $>0, a \leqslant t \leqslant t_{0}$, and $f_{y}\left[u_{R}(t)\right]<0, t_{0} \leqslant t \leqslant b$, Haber and Levinson show that the full problem (1.1), (1.2) has a locally unique ${ }^{2}$ solution $y=y(t, \varepsilon)$ for

Received by the editors November 17, 1976 and, in revised form, February 18, 1977.

AMS (MOS) subject classifications (1970). Primary 34E15; Secondary 34B15.

'Supported by the Department of Mathematics of the University of Wisconsin at Madison and by the National Science Foundation under grant no. MCS 76-05979.

${ }^{2}$ That is, in a sufficiently small neighborhood of $u(t)$ the problem (1.1), (1.2) has only one solution. 
each small $\varepsilon>0$, provided the crossing condition

$$
f\left(t_{0}, u_{L}\left(t_{0}\right), \omega\right) \begin{cases}>0, & u_{L}^{\prime}\left(t_{0}\right)<\omega<u_{R}^{\prime}\left(t_{0}\right), \\ <0, & u_{R}^{\prime}\left(t_{0}\right)<\omega<u_{L}^{\prime}\left(t_{0}\right),\end{cases}
$$

is satisfied. Equally important, the following asymptotic estimates are obtained:

and

$$
\lim _{\varepsilon \rightarrow 0^{+}} y(t, \varepsilon)= \begin{cases}u_{L}(t), & a \leqslant t \leqslant t_{0}, \\ u_{R}(t), & t_{0} \leqslant t \leqslant b,\end{cases}
$$

$$
\lim _{\varepsilon \rightarrow 0^{+}} y^{\prime}(t, \varepsilon)= \begin{cases}u_{L}^{\prime}(t), & a \leqslant t \leqslant t_{0}-\delta, \\ u_{R}^{\prime}(t), & t_{0}+\delta \leqslant t \leqslant b,\end{cases}
$$

for each fixed $\delta>0$ independent of $\varepsilon^{3}$ The results in [4] are valid under mild (i.e., $\left.C^{(1)}\right)$ smoothness restrictions on the function $f=f\left(t, y, y^{\prime}\right)$.

Later writers have almost exclusively concerned themselves with constructing complete asymptotic expansions of the solution of (1.1), (1.2) under basically the same assumptions as in the original formulation of Haber and Levinson. The only additional requirement is that $f$ have sufficiently many derivatives. In reference to such extensions, we note several papers of Vasil'eva [11], [12], [13] and a paper of O'Malley [9]. The results of Vasil'eva actually apply to more general systems than the scalar problem (1.1), (1.2); however, her stability assumptions, specialized to the scalar case, are identical to those of Haber and Levinson, and O'Malley. We remark finally that similar phenomena are studied briefly in the paper of Dorr, Parter and Shampine [3, §5] and in the Russian edition of the book of Wasow [15, §41.4].

In this paper we study generalizations of the basic theorem of Haber and Levinson in a different direction. Namely, we investigate the behavior of solutions of (1.1), (1.2) when the original stability assumptions of [4] are modified or completely absent. Such extensions appear necessary in studying the asymptotic behavior of solutions of problem (1.1), (1.2) in the presence of turning points and/or singular reduced solutions. See, for example, the discussion in [7]. To prove the results stated here, we employ a theorem of Schmitt [10], which can be trivially extended for our purposes. In addition, a theorem of Habets and Laloy [5] is also used. For the convenience of the reader, these theorems are stated in the next section.

2. Two comparison theorems. We state here the two differential inequality results which form the basis for our treatment of (1.1), (1.2). The first is a slight generalization of a theorem, originally proved by Nagumo, and rediscovered by Schmitt [10].

${ }^{3}$ When such a situation obtains we shall say that a Haber-Levinson crossing has occurred. 
Consider the general second-order boundary value problem.

$$
\begin{gathered}
x^{\prime \prime}=F\left(t, x, x^{\prime}\right), \quad a<t<b, \\
x(a)=A, \quad x(b)=B,
\end{gathered}
$$

where $F$ is continuous on $[a, b] \times \mathbf{R}^{2}$.

THEOREM 2.1. Assume there exist continuous functions $\alpha$ and $\beta$ which are piecewise $C^{(2)}$ on $[a, b]$, i.e., there is a finite partition $\left\{t_{i}\right\}, 1 \leqslant i \leqslant n$, of $[a, b]$ suxh that $\alpha$ and $\beta$ are of class $C^{(2)}$ on $\left(t_{i}, t_{i+1}\right)$. In addition, suppose $\alpha(t) \leqslant$ $\beta(t), a \leqslant t \leqslant b, \alpha(a)=A=\beta(a), \alpha(b)=B=\beta(b)$, and $D_{l} \alpha\left(t_{i}\right) \leqslant D_{r} \alpha\left(t_{i}\right)$, $D_{l} \beta\left(t_{i}\right) \geqslant D_{r} \beta\left(t_{i}\right)$, where $D_{l}, D_{r}$ denote lefthand, respectively righthand, differentiation. Assume finally that on $\left(t_{i}, t_{i+1}\right)$,

$$
\alpha^{\prime \prime}(t) \geqslant F\left(t, \alpha(t), \alpha^{\prime}(t)\right) \text { and } \beta^{\prime \prime}(t) \leqslant F\left(t, \beta(t), \beta^{\prime}(t)\right) \text {. }
$$

Then if $\left|F\left(t, x, x^{\prime}\right)\right| \rightarrow \infty$ as $\left|x^{\prime}\right| \rightarrow \infty$, for $(t, x)$ in compact subsets of $[a, b] \times\{\alpha(t) \leqslant x \leqslant \beta(t)\}$, the boundary value problem (2.1), (2.2) has $a$ solution $x=x(t)$ of class $C^{(2)}[a, b]$ with $\alpha(t) \leqslant x(t) \leqslant \beta(t), a \leqslant t \leqslant b$.

This theorem will be used in constructing bounding functions with "corners" at certain points $t_{i}$ in the interior of $[a, b]$ which reflect the angular nature of the solution of the perturbed problem (1.1), (1.2), for small $\varepsilon>0$. Our generalization of the result in [10] is in the spirit of a recent generalization of another thoerem of Nagumo [8] given by Habets and Laloy [5].

THEOREM 2.2. Make the same assumptions as in Theorem 2.1 with the exception that:

(i) the functions $\alpha$ and $\beta$ are only required to satisfy the inequalities $\alpha(a) \leqslant A$ $\leqslant \beta(a)$ and $\alpha(b) \leqslant B<\beta(b)$;

(ii) for $(t, x)$ in $[a, b] \times\{\alpha(t) \leqslant x \leqslant \beta(t)\}$,

$$
F\left(t, x, x^{\prime}\right)=O\left(\left|x^{\prime}\right|^{2}\right), \quad \text { as }\left|x^{\prime}\right| \rightarrow \infty \text {. }
$$

Then the problem (2.1), (2.2) has a solution $x=x(t)$ of class $C^{(2)}[a, b]$ with $\alpha(t) \leqslant x(t) \leqslant \beta(t), a \leqslant t \leqslant b$.

This theorem complements Theorem 2.1 by allowing the function $F$ to have zeros for arbitrarily large values of $x^{\prime}$, provided $F$ is at most quadratic in $x^{\prime}$. We remark finally that, in either theorem, if $\partial f / \partial x$ is strictly positive for $\left(t, x, x^{\prime}\right)$ in $[a, b] \times\{\alpha(t) \leqslant x \leqslant \beta(t)\} \times(-\infty, \infty)$, then the solution $x(t)$ is in fact unique.

3. Modified $f_{y^{\prime}}$-stability. We begin now our study of the possible modifications in the original theory of Haber and Levinson which permit us to deduce the existence of solutions of 


$$
\begin{gathered}
\varepsilon y^{\prime \prime}=f\left(t, y, y^{\prime}\right), \quad a<t<b, \\
y(a, \varepsilon)=A, \quad y(b, \varepsilon)=B,
\end{gathered}
$$

which possess angular limiting behavior at one or several points of $(a, b)$. In the first theorem stated below we only require the sign restrictions on $f_{y}$, $\left[u_{L}\right]$ and $f_{y^{\prime}}\left[u_{R}\right]$ to hold in a small neighborhood of the point of intersection of $u_{L}$ and $u_{R}$. The second result considers the behavior of solutions of (3.1), (3.2) in the presence of a double crossing of reduced solutions of (3.1). Finally we study the occurrence of a Haber-Levinson crossing when one of the reduced solutions is semistable.

\section{THEOREM 3.1. Assume}

(1) the reduced equation $f\left(t, u, u^{\prime}\right)=0$ has two solutions $u=u_{L}(t), u=$ $u_{R}(t)$, defined and of class $C^{(2)}$ on $\left[a, t_{0}\right],\left[t_{0}, b\right]$, respectively, $a<t_{0}<b$, such that $u_{L}(a)=A, u_{L}\left(t_{0}\right)=u_{R}\left(t_{0}\right), u_{L}^{\prime}\left(t_{0}\right) \neq u_{R}^{\prime}\left(t_{0}\right)$, and $u_{R}(b)=B$;

(2) the function $f$ is continuous in all arguments and of class $C^{(2)}$ with respect to $y$ and $y^{\prime}$ in $\Re: a \leqslant t \leqslant b, y-u(t)=O(\varepsilon), y^{\prime}-u^{\prime}(t)=O(d(t, \varepsilon)),{ }^{4}$ where

$$
u(t)=\left\{\begin{array}{ll}
u_{L}(t), & a \leqslant t \leqslant t_{0}, \\
u_{R}(t), & t_{0} \leqslant t \leqslant b,
\end{array} \quad d(t, \varepsilon)=O(\varepsilon),\right.
$$

for $t$ in $[a, b] \backslash\left(t_{0}-\delta, t_{0}+\delta\right), d(t, \varepsilon)=O\left(\left|u_{L}^{\prime}\left(t_{0}\right)-u_{R}^{\prime}\left(t_{0}\right)\right|\right)$, for $t$ in $\left(t_{0}-\right.$ $\left.\delta, t_{0}+\delta\right)$, where $\delta>0$ is a small constant; in addition, $\left|f\left(t, y, y^{\prime}\right)\right| \rightarrow \infty$, as $\left|y^{\prime}\right| \rightarrow \infty$, for $(t, y)$ in $\Re$;

(3) there exists a positive constant $k$ such that $f_{y^{\prime}}\left(t, u_{L}(t), u_{L}^{\prime}(t)\right) \geqslant k>0$, $t_{0}-\delta \leqslant t \leqslant t_{0}$, and $f_{y^{\prime}}\left(t, u_{R}(t), u_{R}^{\prime}(t)\right) \leqslant-k<0, t_{0} \leqslant t \leqslant t_{0}+\delta$, for $\delta>$ 0 the same constant as in (2);

(4) there exists a positive constant $l$ such that $f_{y}\left(t, u_{L}(t), u_{L}^{\prime}(t)\right) \geqslant l>0$, $a \leqslant t \leqslant t_{0}-\delta$, and $f_{y}\left(t, u_{R}(t), u_{R}^{\prime}(t)\right) \geqslant l>0, t_{0}+\delta \leqslant t \leqslant b$;

$$
f\left(t_{0}, u_{L}\left(t_{0}\right), \omega\right)\left\{\begin{array}{ll}
>0 & u_{L}^{\prime}\left(t_{0}\right)<\omega<u_{R}^{\prime}\left(t_{0}\right), \\
<0, & u_{R}^{\prime}\left(t_{0}\right)<\omega<u_{L}^{\prime}\left(t_{0}\right) .
\end{array} \quad\right. \text { (Crossing Condition) }
$$

Then there exists an $\varepsilon_{0}>0$ such that for each $\varepsilon, 0<\varepsilon \leqslant \varepsilon_{0}$, the problem (3.1), (3.2) has a solution $y=y(t, \varepsilon)$. Moreover, if

\footnotetext{
${ }^{4}$ Here and throughout the paper the Landau symbol is defined as: $r(t, \varepsilon)=O(s(t, \varepsilon))$ for $t_{1}<t<t_{2}$ and $0<\varepsilon<\varepsilon_{0}$ iff there exists a constant $C$ (independent of $t$ and $\varepsilon$ ) such that $|r(t, \varepsilon)| \leqslant C|s(t, \varepsilon)|$ for $t_{1}<t<t_{2}$ and $0<\varepsilon<\varepsilon_{0}$.
} 


$$
u(t)= \begin{cases}u_{L}(t), & a \leqslant t \leqslant t_{0}, \\ u_{R}(t), & t_{0} \leqslant t \leqslant b,\end{cases}
$$

then

$$
\begin{gathered}
y(t, \varepsilon)=u(t)+O(\varepsilon), \quad a \leqslant t \leqslant b, \\
y^{\prime}(t, \varepsilon)=u^{\prime}(t)+O\left(\left|u_{L}^{\prime}\left(t_{0}\right)-u_{R}^{\prime}\left(t_{0}\right)\right| \exp \left[-\bar{k}\left|t-t_{0}\right| \varepsilon^{-1}\right]\right)+O(\varepsilon),
\end{gathered}
$$

$a \leqslant t \leqslant b$ for a positive constant $\bar{k}<k$.

Before proving this result we remark that assumption (3) embodies one form of modified $y^{\prime}$-stability in that the positivity (negativity) of $f_{y^{\prime}}$ along $u_{L}\left(u_{R}\right)$ is only required to hold on $\left[t_{0}-\delta, t_{0}\right]\left(\left[t_{0}, t_{0}+\delta\right]\right)$. These sign restrictions have a natural interpretation in that (cf $[12, \S 1]$ ) for $t<t_{0}$ the (linearized) initial value problem

$$
\varepsilon y^{\prime \prime}=f_{y^{\prime}} y^{\prime}+f_{y} y, \quad y\left(t_{0}, \varepsilon\right)=u_{L}\left(t_{0}\right)+O(\varepsilon), \quad y^{\prime}\left(t_{0}, \varepsilon\right)=\omega,
$$

$\omega$ between $u_{L}^{\prime}\left(t_{0}\right)$ and $u_{R}^{\prime}\left(t_{0}\right)$, has a decaying solution provided $f_{y^{\prime}}>0$, while the corresponding problem for $t>t_{0}$ has a decaying solution provided $f_{y^{\prime}}<0$. Finally assumption (4) is a type of $y$-stability assumption (see $\S 4$ below).

Proof. To deduce the existence of a solution $y=y(t, \varepsilon)$ satisfying the first estimate of the theorem, we construct bounding functions $\alpha$ and $\beta$ as described in $\$ 2$ and apply Theorem 2.1. We assume, for definiteness, that $u_{L}^{\prime}\left(t_{0}\right)<u_{R}^{\prime}\left(t_{0}\right)$. The reflected case $u_{L}^{\prime}\left(t_{0}\right)>u_{R}^{\prime}\left(t_{0}\right)$ is handled similarly. Define then for $\varepsilon>0$,

$$
\alpha(t, \varepsilon)= \begin{cases}u_{L}(t)-\sigma(t, \varepsilon), & a \leqslant t \leqslant a+\varepsilon, \\ u_{L}(t)-\varepsilon \gamma l^{-1}, & a+\varepsilon \leqslant t \leqslant t_{0}, \\ u_{R}(t)-\varepsilon \gamma l^{-1}, & t_{0} \leqslant t \leqslant b-\varepsilon, \\ u_{R}(t)-\nu(t, \varepsilon), & b-\varepsilon \leqslant t \leqslant b,\end{cases}
$$

$$
\beta(t, \varepsilon)=\left\{\begin{array}{c}
u_{L}(t)+\sigma(t, \varepsilon), \quad a \leqslant t \leqslant a+\varepsilon, \\
u_{L}(t)+\varepsilon \gamma l^{-1}+w(t, \varepsilon), \quad a+\varepsilon \leqslant t \leqslant t_{0}-\delta, \\
u_{L}(t)-\Gamma_{1} \lambda_{1}^{-1}\left(u_{R}^{\prime}\left(t_{0}\right)-u_{L}^{\prime}\left(t_{0}\right)\right) \exp \left[\lambda_{1}\left(t_{0}-t\right)\right] \\
\quad+\varepsilon \bar{\gamma} L^{-1}\left(\exp \left[-\lambda_{2}\left(t-\left(t_{0}-\delta\right)\right)\right]-1\right), \quad t_{0}-\delta \leqslant t \leqslant t_{0}, \\
u_{R}(t)-\Gamma_{2} \lambda_{1}^{-1}\left(u_{R}^{\prime}\left(t_{0}\right)-u_{L}^{\prime}\left(t_{0}\right)\right) \exp \left[\lambda_{1}\left(t-t_{0}\right)\right] \\
\quad+\varepsilon \bar{\gamma} L^{-1}\left(\exp \left[-\lambda_{2}\left(\left(t_{0}+\delta\right)-t\right)\right]-1\right), \quad t_{0} \leqslant t \leqslant t_{0}+\delta, \\
u_{R}(t)+\varepsilon \gamma l^{-1}+\bar{w}(t, \varepsilon), \quad t_{0}+\delta \leqslant t \leqslant b-\varepsilon, \\
u_{R}(t)+\nu(t, \varepsilon), \quad b-\varepsilon \leqslant t \leqslant b .
\end{array}\right.
$$


Here $\sigma$ is any function satisfying $\sigma \geqslant 0, \sigma=O(\varepsilon), \sigma^{\prime}=O(\varepsilon), \sigma(a, \varepsilon)=0$, $\sigma^{\prime}(a+\varepsilon, \varepsilon) \geqslant 0$ and $\sigma^{\prime \prime} \leqslant-m<0, a \leqslant t \leqslant a+\varepsilon$. Similarly $\nu$ satisfies $\nu \geqslant 0, \nu=O(\varepsilon), \nu^{\prime}=O(\varepsilon), \nu(b, \varepsilon)=0, \nu^{\prime}(b-\varepsilon, \varepsilon) \leqslant 0$ and $\nu^{\prime \prime}<-m<$ $0, b-\varepsilon<t<b$. The functions $w, \bar{w}$ are nonnegative of order $O(\varepsilon)$ and chosen so that $D_{l} \beta>D_{r} \beta$. The constants $\Gamma_{1}, \Gamma_{2}$ are positive and satisfy $\Gamma_{1}+\Gamma_{2}>1$. The quantities $\lambda_{1}, \lambda_{2}$ are the negative $O\left(\varepsilon^{-1}\right)$, respectively, $O(1)$ roots of quadratic polynomials specified below and $L$ is a positive constant such that $\left|f_{y}\left(t, \rho, \rho^{\prime}\right)\right| \leqslant L, \rho=u_{L}$ or $u_{R}, a \leqslant t \leqslant b$. Finally, $\gamma, \bar{\gamma}$ are positive constants to be chosen to make $\alpha$ and $\beta$ well defined ${ }^{5}$ and satisfy the proper inequalities.

It turns out that the quantities $\varepsilon^{-1} \sigma, \varepsilon^{-1} \nu, \gamma$, and $\bar{\gamma}$ must be chosen sufficiently large in order that $\alpha$ and $\beta$ are bounding functions in the sense of Theorem 2.1. Once this is done, these quantities are additionally prescribed to make $\alpha$ and $\beta$ well defined.

We begin by noting that $\alpha<\beta, \alpha(a, \varepsilon)=A=\beta(a, \varepsilon)$, and $\alpha(b, \varepsilon)=B=$ $\beta(b, \varepsilon)$. By the properties of $\sigma, \nu, w, \bar{w}, \Gamma_{1}$ and $\Gamma_{2}, D_{l} \alpha(\hat{t}) \leqslant D_{r} \alpha(\hat{t})$, where $\hat{t}=a+\varepsilon, t_{0}$, and $b-\varepsilon$, and $D_{l} \beta(\bar{t}) \geqslant D_{r} \beta(\bar{t})$, where $\bar{t}=a+\varepsilon, t_{0}-\delta, t_{0}$, $t_{0}+\delta$, and $b-\varepsilon$. To verify that the differential inequalities are satisfied, we need only check each of the subintervals $(a, a+\varepsilon), \ldots,(b-\varepsilon, b)$. For example, consider $\alpha(t, \varepsilon)$ defined on $\left(a+\varepsilon, t_{0}\right)$. The function $f\left(t, \alpha, \alpha^{\prime}\right)$ may be written as

$$
\begin{aligned}
f\left(t, \alpha, \alpha^{\prime}\right)= & f\left(t, u_{L}, u_{L}^{\prime}\right)+\left\{f\left(t, \alpha, u_{L}^{\prime}\right)-f\left(t, u_{L}, u_{L}^{\prime}\right)\right\} \\
& +\left\{f\left(t, \alpha, \alpha^{\prime}\right)-f\left(t, \alpha, u_{L}^{\prime}\right)\right\} \\
= & f\left(t, u_{L}, u_{L}^{\prime}\right)+f_{y}\left(t, u_{L}+\theta_{1}\left(\alpha-u_{L}\right), u_{L}^{\prime}\right)\left(\alpha-u_{L}\right) \\
& +f_{y^{\prime}}\left(t, \alpha, u_{L}^{\prime}+\theta_{2}\left(\alpha^{\prime}-u_{L}^{\prime}\right)\right)\left(\alpha^{\prime}-u_{L}^{\prime}\right), \quad 0<\theta_{1}, \theta_{2}<1 .
\end{aligned}
$$

Differentiating $\alpha$ and substituting into this expansion, we have for small $\varepsilon>0$,

$$
\begin{aligned}
\varepsilon \alpha^{\prime \prime}-f\left(t, \alpha, \alpha^{\prime}\right) & =\varepsilon u_{L}^{\prime \prime}-f\left(t, u_{L}, u_{L}^{\prime}\right)+f_{y}[t, \varepsilon] \varepsilon \gamma l^{-1} \\
& \geqslant-\varepsilon M+\varepsilon \gamma \geqslant 0,
\end{aligned}
$$

if $\gamma$ is chosen greater than $M$. Here $[t, \varepsilon]$ is the intermediate point and $\left|u_{L}^{\prime \prime}\right| \leqslant M$.

Similarly, on $(b-\varepsilon, b)$,

$$
\begin{aligned}
\varepsilon \alpha^{\prime \prime}-f\left(t, \alpha, \alpha^{\prime}\right) & =\varepsilon u_{R}^{\prime \prime}-\varepsilon \nu^{\prime \prime}-f\left(t, u_{R}, u_{R}^{\prime}\right)+f_{y}[t, \varepsilon] \nu+f_{y^{\prime}}\{t, \varepsilon\} \nu^{\prime} \\
& \geqslant-\varepsilon M-\varepsilon \nu^{\prime \prime}+l \nu-k \nu^{\prime} \geqslant 0,
\end{aligned}
$$

if $-\nu^{\prime \prime}$ is chosen greater than $M+\varepsilon^{-1} k \nu^{\prime}$. (Recall that $\nu^{\prime}=O(\varepsilon)$ and

\footnotetext{
${ }^{5}$ That is, $\eta(a+\varepsilon), \eta\left(t_{0}-\delta\right), \eta\left(t_{0}\right), \eta\left(t_{0}+\delta\right)$ and $\eta(b-\varepsilon)$, for $\eta=\alpha$ or $\beta$, are uniquely determined.
} 
$\nu^{\prime \prime} \leqslant-m<0$.) The verification that $\alpha$ satisfies this inequality on $(a, a+\varepsilon)$ and $\left(t_{0}, b-\varepsilon\right)$ proceeds analogously.

As for $\beta$, we only need to see that the correct inequality is satisfied, for example, on $\left(t_{0}-\delta, t_{0}\right)$, since the other intervals are handled in basically the same way. First consider the subinterval $\left[t_{0}-\tau \varepsilon, t_{0}\right], \tau>0$ and $\tau=O(1){ }^{6}$ Then for $\varepsilon$ sufficiently small, we invoke the differentiability of $f$ to see that

$$
\begin{aligned}
f\left(t, \beta, \beta^{\prime}\right)-\varepsilon \beta^{\prime \prime}= & f\left(t, u_{L}, u_{L}^{\prime}\right) \\
+ & +f_{y}[t, \varepsilon]\left\{-\Gamma_{1} \lambda_{1}^{-1}\left(u_{R}^{\prime}\left(t_{0}\right)-u_{L}^{\prime}\left(t_{0}\right)\right) \cdot \exp \left[\lambda_{1}\left(t_{0}-t\right)\right]\right. \\
& \left.+\varepsilon \gamma L^{-1}\left(\exp \left[-\lambda_{2}\left(t-\left(t_{0}-\tau \varepsilon\right)\right)\right]-1\right)\right\} \\
& +f_{y^{\prime}}\left(t, \beta, u_{L}^{\prime}\right)\left\{\Gamma_{1}\left(u_{R}^{\prime}\left(t_{0}\right)-u_{L}^{\prime}\left(t_{0}\right)\right) \cdot \exp \left[\lambda_{1}\left(t_{0}-t\right)\right]\right. \\
& \left.-\lambda_{2} \varepsilon \gamma L^{-1} \exp \left[-\lambda_{2}\left(t-\left(t_{0}-\tau \varepsilon\right)\right)\right]\right\} \\
+ & \frac{1}{2} f_{y^{\prime} y^{\prime}}\{t, \varepsilon\}\left\{\Gamma_{1}\left(u_{R}^{\prime}\left(t_{0}\right)-u_{L}^{\prime}\left(t_{0}\right)\right) \cdot \exp \left[\lambda_{1}\left(t_{0}-t\right)\right]\right. \\
& \left.\quad-\lambda_{2} \varepsilon \gamma L^{-1} \exp \left[-\lambda_{2}\left(t-\left(t_{0}-\tau \varepsilon\right)\right)\right]\right\}^{2} \\
& -\varepsilon u_{L}^{\prime \prime}+\varepsilon \lambda_{1} \Gamma_{1}\left(u_{R}^{\prime}\left(t_{0}\right)-u_{L}^{\prime}\left(t_{0}\right)\right) \exp \left[\lambda_{1}\left(t_{0}-t\right)\right] \\
& -\varepsilon \lambda_{2}^{2} \varepsilon \gamma L^{-1} \exp \left[-\lambda_{2}\left(t-\left(t_{0}-\tau \varepsilon\right)\right)\right] .
\end{aligned}
$$

By assumption (5) $f\left(t_{0}, u_{L}\left(t_{0}\right), \omega\right)>0, u_{\mathrm{L}}^{\prime}\left(t_{0}\right)<\omega<u_{R}^{\prime}\left(t_{0}\right)$, which is equivalent to

$$
\begin{aligned}
& f_{y^{\prime}}\left(t_{0}, u_{L}\left(t_{0}\right), u_{L}^{\prime}\left(t_{0}\right)\right)+\frac{1}{2} f_{y^{\prime} y^{\prime}}\left(t_{0},\right.\left.u_{L}\left(t_{0}\right), u_{L}^{\prime}\left(t_{0}\right)+\theta\left(\beta^{\prime}-u_{L}^{\prime}\right)\left(t_{0}\right)\right) \\
& \cdot\left(u_{R}^{\prime}\left(t_{0}\right)-u_{L}^{\prime}\left(t_{0}\right)\right) \exp \left[\lambda_{1}\left(t_{0}-t\right)\right] \geqslant K>0 .
\end{aligned}
$$

We can continue then with the inequality

$$
\begin{aligned}
f\left(t, \beta, \beta^{\prime}\right)- & \varepsilon \beta^{\prime \prime} \geqslant\left(u_{R}^{\prime}\left(t_{0}\right)-u_{L}^{\prime}\left(t_{0}\right)\right) \exp \left[\lambda_{1}\left(t_{0}-t\right)\right] \\
& \cdot\left\{L \Gamma_{1} \lambda_{1}^{-1}+\bar{k} \Gamma_{1}+\varepsilon \lambda_{1} \Gamma_{1}\right\} \\
& -\exp \left[-\lambda_{2}\left(t-\left(t_{0}-\tau \varepsilon\right)\right)\right]\left\{\varepsilon \gamma+k \lambda_{2} \varepsilon \gamma L^{-1}+\varepsilon \lambda_{2}^{2} \varepsilon \gamma L^{-1}\right\} \\
+ & \varepsilon \gamma-\varepsilon\left|u_{L}^{\prime \prime}\right|-N(\gamma) \varepsilon^{2} .
\end{aligned}
$$

Here $N(\gamma) \geqslant \frac{1}{2}\left|f_{y^{\prime} y^{\prime}}\{t, \varepsilon\}\right| \lambda_{2}^{2} \gamma^{2} L^{-2} \exp \left[-2 \lambda_{2} \tau \varepsilon\right]$. Now choose $\lambda_{1}$ as the $O\left(\varepsilon^{-1}\right)$ (negative) root of $\varepsilon \lambda^{2}+\bar{k} \lambda+L=0$ where $0<\bar{k}<\min \{k, K\}$ and $\lambda_{2}$ as the $O(1)$ (negative) root of $\varepsilon \lambda^{2}+k \lambda+L=0$. Then we have finally that $f\left(t, \beta, \beta^{\prime}\right)-\varepsilon \beta^{\prime \prime} \geqslant \varepsilon \gamma-\varepsilon M-N(\gamma) \varepsilon^{2}>0$ for $t$ in $\left[t_{0}-\tau \varepsilon, t_{0}\right]$ if $\gamma=M+$ 1 and $\varepsilon$ is sufficiently small.

On the subinterval $\left[t_{0}-\delta, t_{0}-\tau \varepsilon\right]$ for $\tau$ sufficiently large we have

${ }^{6}$ The estimates which follow hold uniformly for $\tau$ in the range $0<\tau_{0}<\tau<\tau_{1}<\infty$. 


$$
f_{y^{\prime}}[t, \varepsilon]=f_{y^{\prime}}\left(t, u_{L}(t), u_{L}^{\prime}(t)\right)+O\left(\varepsilon^{2}\right)
$$

where $[t, \varepsilon]=\left(t, u_{L}(t), \beta^{\prime}(t, \varepsilon)\right)$.

Clearly then

$$
\begin{aligned}
f\left(t, \beta, \beta^{\prime}\right)-\varepsilon \beta^{\prime \prime}= & f\left[u_{L}\right]+\left\{f\left(t, \beta, u_{L}^{\prime}\right)-f\left(t, u_{L}, u_{L}^{\prime}\right)\right\} \\
& +\left\{f\left(t, \beta, \beta^{\prime}\right)-f\left(t, \beta, u_{L}^{\prime}\right)\right\} \\
\geqslant & L \Gamma_{1} \lambda_{1}^{-1}\left(u_{R}^{\prime}\left(t_{0}\right)-u_{L}^{\prime}\left(t_{0}\right)\right) \exp \left[\lambda_{1}\left(t_{0}-t\right)\right] \\
& -\varepsilon \gamma \exp \left[-\lambda_{2} t-\left(t_{0}-\delta\right)\right]+\varepsilon \gamma \\
& +k \Gamma_{1}\left(u_{R}^{\prime}\left(t_{0}\right)-u_{L}^{\prime}\left(t_{0}\right)\right) \exp \left[\lambda_{1}\left(t_{0}-t\right)\right] \\
& -k \lambda_{2} \varepsilon \gamma L^{-1} \exp \left[-\lambda_{2}\left(t-\left(t_{0}-\delta\right)\right)\right] \\
& -\varepsilon M+\varepsilon \lambda_{1} \Gamma_{1}\left(u_{R}^{\prime}\left(t_{0}\right)-u_{L}^{\prime}\left(t_{0}\right)\right) \exp \left[\lambda_{1}\left(t_{0}-t\right)\right] \\
& -\varepsilon \lambda_{2}^{2} \varepsilon \gamma L^{-1} \exp \left[-\lambda_{2}\left(t-\left(t_{0}-\delta\right)\right)\right] \\
= & -\varepsilon M+\varepsilon \gamma \geqslant 0, \text { for } \gamma \geqslant M .
\end{aligned}
$$

Here we have used the fact that $\lambda_{1}$ and $\lambda_{2}$ are roots of $\varepsilon \lambda^{2}+\bar{k} \lambda+L=0$, $0<\bar{k} \leqslant k$. We conclude by Theorem 2.1 that for each $\varepsilon>0, \varepsilon$ sufficiently small, the problem (3.1), (3.2) has a solution $y=y(t, \varepsilon)$ such that $\alpha(t, \varepsilon) \leqslant$ $y(t, \varepsilon) \leqslant \beta(t, \varepsilon)$, i.e., $y(t, \varepsilon)=u(t)+O(\varepsilon), a \leqslant t \leqslant b$. To derive the estimate on $y^{\prime}-u^{\prime}$, we make the substitution $z=y-u$ in (3.1), (3.2) and obtain a representation for $z^{\prime}$, i.e., for $t$ in $\left(a, t_{0}\right)$,

$$
\varepsilon z^{\prime \prime}=f_{y^{\prime}}\{t, \varepsilon\}_{L^{\prime}} z^{\prime}+f_{y}\{t, \varepsilon\}_{L^{\prime}} z-\varepsilon u_{L}^{\prime \prime}(t)=f_{y^{\prime}}\{t, \varepsilon\}_{L^{\prime}} z^{\prime}+O(\varepsilon)
$$

and for $t$ in $\left(t_{0}, b\right)$,

$$
\varepsilon z^{\prime \prime}=f_{y^{\prime}}\{t, \varepsilon\}_{R^{\prime}} z^{\prime}+f_{y}\{t, \varepsilon\}_{R^{z}}-\varepsilon u_{R}^{\prime \prime}(t)=f_{y^{\prime}}\{t, \varepsilon\}_{R^{\prime}} z^{\prime}+O(\varepsilon) .
$$

Here $\{t, \varepsilon\}_{L, R}$ are the appropriate intermediate points. Consider now just the subinterval $\left(a, t_{0}\right)$; the analogous argument for $\left(t_{0}, b\right)$ is omitted. On $\left(a, t_{0}-\right.$ ס) $z$ satisfies

$$
\begin{gathered}
\varepsilon z^{\prime \prime}=f_{y^{\prime}}\{t, \varepsilon\}_{L^{2}} z^{\prime}+O(\varepsilon), \quad a<t<t_{0}-\delta, \\
z(a, \varepsilon)=0, \quad z\left(t_{0}-\delta\right)=O(\varepsilon),
\end{gathered}
$$

and it follows from the maximum principle (cf., e.g., [3]) that $z^{\prime}(t, \varepsilon)=O(\varepsilon)$, $a \leqslant t \leqslant t_{0}-\delta$. On $\left(t_{0}-\delta, t_{0}\right) z$ satisfies

$$
\begin{aligned}
\varepsilon z^{\prime \prime} & =f_{y^{\prime}}\{t, \varepsilon\}_{L^{\prime}} z^{\prime}+O(\varepsilon), \quad t_{0}-\delta<t<t_{0}, \\
z\left(t_{0}, \varepsilon\right) & =O(\varepsilon), \quad z^{\prime}\left(t_{0}, \varepsilon\right)=O\left(\left|u_{L}^{\prime}\left(t_{0}\right)-u_{R}^{\prime}\left(t_{0}\right)\right|\right) .
\end{aligned}
$$

But assumptions (3) and (5) imply that for such $t f_{y^{\prime}}\{t, \varepsilon\}_{L} \geqslant \tilde{k}>0$ and so the solution of this initial value problem satisfies 


$$
\begin{aligned}
z^{\prime}(t, \varepsilon)=O\left(\left|u_{L}^{\prime}\left(t_{0}\right)-u_{R}^{\prime}\left(t_{0}\right)\right| \exp \left[\tilde{k}\left(t-t_{0}\right) \varepsilon^{-1}\right]\right) & +O(\varepsilon), \\
t_{0} & -\delta \leqslant t \leqslant t_{0} .
\end{aligned}
$$

(cf. $[12, \S 1])$.

In the next theorem we examine conditions under which a double HaberLevinson crossing can occur. The extension to an arbitrary number of finite crossings is obvious.

\section{THEOREM 3.2. Assume}

(1) the reduced equation $f\left(t, u, u^{\prime}\right)=0$ has three solutions $u=u_{L}(t), u=$ $u_{M}(t), u=u_{R}(t)$, defined and of class $C^{(2)}$ on $\left[a, t_{1}\right],\left[t_{1}, t_{2}\right],\left[t_{2}, b\right]$, respectively, $a<t_{1}<t_{2}<b$, with $u_{L}(a)=A, u_{L}\left(t_{1}\right)=u_{M}\left(t_{1}\right), u_{M}\left(t_{2}\right)=u_{R}\left(t_{2}\right)$ and $u_{R}(b)$ $=B$; in addition, $u_{L}^{\prime}\left(t_{1}\right) \neq u_{M}^{\prime}\left(t_{1}\right)$ and $u_{M}^{\prime}\left(t_{2}\right) \neq u_{R}^{\prime}\left(t_{2}\right)$;

(2) the function $f$ is continuous in all arguments and of class $C^{(2)}$ with respect to $y$ and $y^{\prime}$ in $\mathscr{D}: a \leqslant t \leqslant b, y-u(t)=O(\varepsilon), y^{\prime}-u^{\prime}(t)=O(d(t, \varepsilon))$, where

$$
u(t)= \begin{cases}u_{L}(t), & a \leqslant t \leqslant t_{1}, \\ u_{M}(t), & t_{1} \leqslant t \leqslant t_{2}, \\ u_{R}(t), & t_{2} \leqslant t \leqslant b,\end{cases}
$$

and $d(t, \varepsilon)=O(\varepsilon)$, for $t$ in $[a, b] \backslash\left\{\left(t_{1}-\delta, t_{1}+\delta\right) \cup\left(t_{2}-\delta, t_{2}+\delta\right)\right\}$, $d(t, \varepsilon)=O\left(\left|u_{L}^{\prime}\left(t_{1}\right)-u_{M}^{\prime}\left(t_{1}\right)\right|\right)$, for $t$ in $\left(t_{1}-\delta, t_{1}+\delta\right)$, and $d(t, \varepsilon)=$ $O\left(\left|u_{M}^{\prime}\left(t_{2}\right)-u_{R}^{\prime}\left(t_{2}\right)\right|\right)$, for $t$ in $\left(t_{2}-\delta, t_{2}+\delta\right)$; here $\delta>0$ is a small constant; in addition, $\left|f\left(t, y, y^{\prime}\right)\right| \rightarrow \infty$ as $\left|y^{\prime}\right| \rightarrow \infty$, for $(t, y)$ in $\mathscr{W}$;

(3) there exists a positive constant $k$ such that

$$
\begin{aligned}
f_{y^{\prime}}\left(t, u_{L}(t), u_{L}^{\prime}(t)\right) & \geqslant k>0, \quad a \leqslant t \leqslant t_{1}, \\
f_{y^{\prime}}\left(t, u_{M}(t), u_{M}^{\prime}(t)\right) & \leqslant-k<0, \quad t_{1} \leqslant t \leqslant t_{1}+\delta, \\
f_{y^{\prime}}\left(t, u_{M}(t), u_{M}^{\prime}(t)\right) & \geqslant k>0, \quad t_{2}-\delta \leqslant t \leqslant t_{2}, \\
f_{y^{\prime}}\left(t, u_{R}(t), u_{R}^{\prime}(t)\right) & \leqslant-k<0, \quad t_{2} \leqslant t \leqslant 1 ;
\end{aligned}
$$

(4) there exists a positive constant $l$ such that

$$
\begin{gathered}
f_{y}\left(t, u_{M}(t), u_{M}^{\prime}(t)\right) \geqslant l>0, \quad t_{1}+\delta \leqslant t \leqslant t_{2}-\delta ; \\
f\left(t_{1}, u_{L}\left(t_{1}\right), \omega\right) \begin{cases}>0, & u_{L}^{\prime}\left(t_{1}\right)<\omega<u_{M}^{\prime}\left(t_{1}\right), \\
<0, & u_{M}^{\prime}\left(t_{1}\right)<\omega<u_{L}^{\prime}\left(t_{1}\right),\end{cases}
\end{gathered}
$$

and

$$
f\left(t_{2}, u_{R}\left(t_{2}\right), \omega\right) \begin{cases}>0, & u_{M}^{\prime}\left(t_{2}\right)<\omega<u_{R}^{\prime}\left(t_{2}\right), \\ <0, & u_{R}^{\prime}\left(t_{2}\right)<\omega<u_{M}^{\prime}\left(t_{2}\right) .\end{cases}
$$

Then there exists an $\varepsilon_{0}>0$ such that for each $\varepsilon, 0<\varepsilon \leqslant \varepsilon_{0}$, the problem (3.1), (3.2) has a solution $y=y(t, \varepsilon)$. In addition, for 


$$
\begin{aligned}
& u(t)= \begin{cases}u_{L}(t), & a \leqslant t \leqslant t_{1}, \\
u_{M}(t), & t_{1} \leqslant t \leqslant t_{2}, \\
u_{R}(t), & t_{2} \leqslant t \leqslant b,\end{cases} \\
& y(t, \varepsilon)=u(t)+O(\varepsilon), \quad a \leqslant t \leqslant b, \quad \text { and } \\
& y^{\prime}(t, \varepsilon)=u^{\prime}(t)+O\left(\left|u_{L}^{\prime}\left(t_{1}\right)-u_{M}^{\prime}\left(t_{1}\right)\right| \exp \left[-\bar{k}\left|t-t_{1}\right| \varepsilon^{-1}\right]\right) \\
& +O\left(\left|u_{R}^{\prime}\left(t_{2}\right)-u_{M}^{\prime}\left(t_{2}\right)\right| \exp \left[-\bar{k}\left|t-t_{2}\right| \varepsilon^{-1}\right]\right)+O(\varepsilon), \\
& a \leqslant t \leqslant b,
\end{aligned}
$$

where $0<\bar{k}<k$.

Proof. We proceed essentially as in the proof of Theorem 3.1. Suppose, for definiteness, that $u_{L}^{\prime}\left(t_{1}\right)<u_{M}^{\prime}\left(t_{1}\right)$ and $u_{M}^{\prime}\left(t_{2}\right)>u_{R}^{\prime}\left(t_{2}\right)$. Define for $\varepsilon>0$,

$$
\alpha(t, \varepsilon)=\left\{\begin{array}{c}
u_{L}(t)-\gamma_{1} L^{-1}\left(\exp \left[-\lambda_{2}(t-a)\right]-1\right), \quad a<t \leqslant t_{1}, \\
u_{M}(t)-\varepsilon \gamma_{2} L^{-1}\left(\exp \left[-\lambda_{2}\left(\left(t_{1}+\delta\right)-t\right)\right]-1\right), \quad t_{1}<t<t_{1}+\delta, \\
u_{M}(t)-\varepsilon \gamma_{3} l^{-1}-w(t, \varepsilon), t_{1}+\delta \leqslant t \leqslant t_{2}-\delta, \\
u_{M}(t)+\Gamma_{3} \lambda_{1}^{-1}\left(u_{M}^{\prime}\left(t_{2}\right)-u_{R}^{\prime}\left(t_{2}\right)\right) \exp \left[\lambda_{1}\left(t_{2}-t\right)\right] \\
\quad-\varepsilon \gamma_{4} L^{-1}\left(\exp \left[-\lambda_{2}\left(t-\left(t_{2}-\delta\right)\right)\right]-1\right), \quad t_{2}-\delta \leqslant t<t_{2}, \\
u_{R}(t)+\Gamma_{4} \lambda_{1}^{-1}\left(u_{M}^{\prime}\left(t_{2}\right)-u_{R}^{\prime}\left(t_{2}\right)\right) \exp \left[\lambda_{1}\left(t-t_{2}\right)\right] \\
-\varepsilon \gamma_{5} L^{-1}\left(\exp \left[\lambda_{2}(t-b)\right]-1\right), \quad t_{2}<t<b,
\end{array}\right.
$$

and

$$
\beta(t, \varepsilon)=\left\{\begin{aligned}
& u_{L}(t)-\Gamma_{1} \lambda_{1}^{-1}\left(u_{M}^{\prime}\left(t_{1}\right)-u_{L}^{\prime}\left(t_{1}\right)\right) \exp \left[\lambda_{1}\left(t_{1}-t\right)\right] \\
&+\varepsilon \bar{\gamma}_{1} L^{-1}\left(\exp \left[-\lambda_{2}(t-a)\right]-1\right), \quad a<t<t_{1}, \\
& u_{M}(t)-\Gamma_{2} \lambda_{1}^{-1}\left(u_{M}^{\prime}\left(t_{1}\right)-u_{L}^{\prime}\left(t_{1}\right)\right) \exp \left[\lambda_{1}\left(t-t_{1}\right)\right] \\
&+\varepsilon \bar{\gamma}_{2} L^{-1}\left(\exp \left[\lambda_{2}\left(t-\left(t_{1}+\delta\right)\right)\right]-1\right), \quad t_{1}<t \leqslant t_{1}+\delta, \\
& u_{M}(t)+\varepsilon \bar{\gamma}_{3} l^{-1}+w(t, \varepsilon), t_{1}+\delta \leqslant t \leqslant t_{2}-\delta, \\
& u_{M}(t)+\varepsilon \bar{\gamma}_{4} L^{-1}\left(\exp \left[-\lambda_{2}\left(t-\left(t_{2}-\delta\right)\right)\right]-1\right), \quad t_{2}-\delta \leqslant t<t_{2}, \\
& u_{R}(t)+\varepsilon \bar{\gamma}_{5} L^{-1}\left(\exp \left[\lambda_{2}(t-b)\right]-1\right), \quad t_{2}<t<b .
\end{aligned}\right.
$$

It is easy to see that $\alpha \leq \beta, \alpha(a, \varepsilon)=A=\beta(a, \varepsilon), \alpha(b, \varepsilon)=B=\beta(b, \varepsilon)$, and that the remaining differential inequalities are satisfied. Here $\lambda_{1}, \lambda_{2}$ are the $O\left(\varepsilon^{-1}\right), O(1)$ roots of $\varepsilon \lambda^{2}+k \lambda+L=0$ and $w(t, \varepsilon)$ is as described in the proof of Theroem 3.1. 
These two results just given present on the one hand sufficient conditions for solutions of the problem (3.1), (3.2) to possess Haber-Levinson behavior under modified assumptions on the solutions of the reduced equation $f\left(t, u, u^{\prime}\right)=0$. On the other hand, they can also be interpreted as demonstrating that reduced solutions can have several types of stability, namely, the classical $f_{y}$-stability of assumption (3) as well as the $f_{y}$-stability of assumption (4). In the next section we study types of stability other than $f_{y}$-stability, and achieve essentially the same results.

The final situation we consider in this section involves a solution of the reduced equation, $u_{L}$ or $u_{R}$, which is semistable in a neighborhood of the point of intersection $t_{0}$ of $u_{L}$ and $u_{R}$, i.e., $f_{y^{\prime}}\left[u_{L}(t)\right] \geqslant 0$ or $f_{y^{\prime}}\left[u_{R}(t)\right] \leqslant 0$, for $t$ near $t_{0}$. Suppose, for example, that $u_{L}$ is semistable, but that $f_{y}\left[u_{L}(t)\right]$ is positive, then if $u_{R}$ is stable, i.e., $f_{y}\left[u_{R}(t)\right]<0$, and if the crossing condition is satisfied at $t_{0}$, it is reasonable to expect that a Haber-Levinson crossing occurs at $t_{0}$. This follows because the semistability of $u_{L}$ and the $y$-stability implied by the assumption that $f_{y}\left[u_{L}\right]>0$ combine to allow $u_{L}$ to attract a solution of (3.1), (3.2) for small $\varepsilon>0$. The precise result is the following theorem.

Theorem 3.3. Assume (1), (2), (4) and (5) as in Theorem 3.1. Assume either

(3) there exists a positive constant $k$ such that

$$
\begin{aligned}
& f_{y^{\prime}}\left(t, u_{L}(t), u_{L}^{\prime}(t)\right) \geqslant k>0, \quad t_{0}-\delta \leqslant t \leqslant t_{0}, \quad \text { and } \\
& f_{y^{\prime}}\left(t, u_{R}(t), u_{R}^{\prime}(t)\right) \leqslant 0, \quad t_{0} \leqslant t \leqslant t_{0}+\delta ;
\end{aligned}
$$

in addition,

$$
f_{y}\left(t, u_{R}(t), u_{R}^{\prime}(t)\right) \geqslant l>0, t_{0} \leqslant t \leqslant t_{0}+\delta,
$$

for the same constant $l$ in (4); or

(3)' there exists a positive constant $k$ such that

$$
\begin{aligned}
& f_{y^{\prime}}\left(t, u_{R}(t), u_{R}^{\prime}(t)\right) \leqslant-k<0, \quad t_{0} \leqslant t \leqslant t_{0}+\delta, \quad \text { and } \\
& f_{y^{\prime}}\left(t, u_{L}(t), u_{L}^{\prime}(t)\right) \geqslant 0, \quad t_{0}-\delta \leqslant t \leqslant t_{0} ;
\end{aligned}
$$

in addition,

$$
f_{y}\left(t, u_{L}(t), u_{L}^{\prime}(t)\right) \geqslant l>0, \quad t_{0}-\delta \leqslant t \leqslant t_{0} .
$$

Then there exists an $\varepsilon_{0}>0$ such that for each $\varepsilon, 0<\varepsilon \leqslant \varepsilon_{0}$, the problem (3.1), (3.2) has a solution $y=y(t, \varepsilon)$ which satisfies for

$$
\begin{aligned}
u(t) & = \begin{cases}u_{L}(t), & a \leqslant t \leqslant t_{0}, \\
u_{R}(t), & t_{0} \leqslant t \leqslant b,\end{cases} \\
y(t, \varepsilon) & =u(t)+O\left(\varepsilon^{1 / 2}\right), \quad a \leqslant t \leqslant b .
\end{aligned}
$$

This theorem is proved in basically the same manner as Theorem 3.1. The only important exception is that the layer correction term is a function of $t$ 
and $\varepsilon^{1 / 2}$, and it depends on whether (3) or (3)' holds. For example, if

$$
f_{y^{\prime}}\left[u_{L}(t)\right] \geqslant 0, \quad t_{0}-\delta \leqslant t \leqslant t_{0},
$$

then the layer term is of the form $\varepsilon^{1 / 2} \exp \left[\left(\varepsilon^{-1} l\right)^{1 / 2}\left(t-t_{0}\right)\right]$ and it is added to $u_{L}$. The order term $\varepsilon^{1 / 2}$ is required since $f_{y^{\prime}}\left[u_{L}(t)\right]$ may be zero at or near $t=t_{0}$, and consequently, the layer term has to satisfy $\varepsilon y^{\prime \prime}=l y$, where $f_{y} \geqslant l>0$.

We conclude this section with several examples.

EXAMPLE 3.1. Consider the problem

$$
\begin{array}{rlrl}
\varepsilon y^{\prime \prime} & =y-2 t y^{\prime}+y^{\prime 2}, & -1<t<1, \\
y(-1, \varepsilon) & =A, & y(1, \varepsilon)=\frac{3}{4}, & 0<A<\frac{3}{4} .
\end{array}
$$

The stable solution of the reduced equation satisfying the righthand boundary condition is $u_{R}(t)=\frac{3}{4} t^{2}$, while the stable reduced solution $u_{L}$ satisfying $u_{L}(-1)=A$ is given parametrically by

$$
t=\frac{2}{3} \tau+C \tau^{-2}, \quad u_{L}=2 t \tau-\tau^{2}, \quad \sqrt[3]{3 C}<\tau<0,
$$

for some constant $C<0$. The functions $u_{L}$ and $u_{R}$ intersect at a point $t_{0}$ in $(-1,0)$ with $u_{L}^{\prime}\left(t_{0}\right)=t_{0} / 2>u_{R}^{\prime}\left(t_{0}\right)=\frac{3}{2} t_{0}$. These solutions are stable in the sense that $f_{y^{\prime}}\left[u_{L}(t)\right]=2 u_{L}^{\prime}-2 t=2(\tau-t)=-\frac{2}{3} \tau+2 C \tau^{-2}>0,-1 \leqslant t$ $\leqslant t_{0}$, and $f_{y}\left[u_{R}(t)\right]=t, t_{0} \leqslant t \leqslant 1$. It only remains to see that the crossing condition is satisfied, i.e.,

$$
f\left(t_{0}, u_{L}\left(t_{0}\right), \omega\right)=\omega^{2}-2 t_{0} \omega+\frac{3}{4} t_{0}^{2}=\left(\omega-\frac{3}{2} t_{0}\right)\left(\omega-\frac{1}{2} t_{0}\right)<0,
$$

for $\frac{3}{2} t_{0}<\omega<\frac{1}{2} t_{0}$. Since $f_{y} \equiv 1$, we deduce from Theorem 3.1 that the problem has a unique solution $y=y(t, \varepsilon)$ such that

$$
\begin{aligned}
& y(t, \varepsilon)=u_{L}(t)+O(\varepsilon), \quad-1 \leqslant t \leqslant t_{0}, \quad \text { and } \\
& y(t, \varepsilon)=\frac{3}{4} t^{2}+O(\varepsilon), \quad t_{0} \leqslant t \leqslant 1 .
\end{aligned}
$$

EXAMPLE 3.2. Consider next the problem

$$
\begin{gathered}
\varepsilon y^{\prime \prime}=y^{\prime 2}-2 t y^{\prime}, \quad-1<t<1, \\
y(-1, \varepsilon)=A, y(1, \varepsilon)=B, \quad A-1<B<A+1, A \neq B .
\end{gathered}
$$

The relevant solutions of the reduced equation are

$$
\begin{aligned}
& u_{L}(t)=\left\{\begin{array}{ll}
A, & -1 \leqslant t \leqslant 0, \\
t^{2}+A, & 0 \leqslant t \leqslant 1,
\end{array}\right. \text { and } \\
& u_{R}(t)= \begin{cases}t^{2}+B, & -1 \leqslant t \leqslant 0, \\
B, & 0 \leqslant t \leqslant 1 .\end{cases}
\end{aligned}
$$

Although $u_{L}, u_{R}$ are not of class $C^{(2)}[-1,1], D_{l, r}\left\{u_{L}^{\prime}, u_{R}^{\prime}\right\}$ exist and are bounded, so the above theory applies with no real modification. For our choice of $A$ and $B, u_{L}\left(t_{0}\right)=u_{R}\left(t_{0}\right)$ and $u_{L}^{\prime}\left(t_{0}\right) \neq u_{R}^{\prime}\left(t_{0}\right)$, where $t_{0}=-(A-$ 
$B)^{1 / 2}$, if $0<A-B<1$, and $t_{0}=(B-A)^{1 / 2}$, if $0<B-A<1$. The stability assumptions of Theorem 3.1 are satisfied since $f_{y}\left[u_{L}(t)\right]=2|t|$ and $f_{y}\left[u_{R}(t)\right]=-2|t|$; in addition, the crossing condition is also satisfied. Theorem 3.1 is not however directly applicable since $f_{y} \equiv 0$. We can nevertheless construct bounding solutions similar to those above and deduce the existence of a solution $y=y(t, \varepsilon)$ such that

$$
\begin{array}{ll}
y(t, \varepsilon)=u_{L}(t)+O(\varepsilon), & -1 \leqslant t \leqslant t_{0}, \quad \text { and } \\
y(t, \varepsilon)=u_{R}(t)+O(\varepsilon), & t_{0} \leqslant t \leqslant 1 .
\end{array}
$$

EXAMPLE 3.3. The problem of Example $3.1 \mathrm{can}$ be used to illustrate the phenomenon of a double crossing treated in Theorem 3.2 by changing slightly the boundary conditions. Consider

$$
\begin{aligned}
\varepsilon y^{\prime \prime} & =y-2 t y^{\prime}+y^{\prime 2}, \quad-1<t<1, \\
y(-1, \varepsilon) & =A, \quad y(1, \varepsilon)=B, \quad 0<A, B<\frac{3}{4} .
\end{aligned}
$$

The solution $u_{L}$ is given parametrically by $t=\frac{2}{3} \tau+c_{1} \tau^{-2}, u_{L}=2 t \tau-\tau^{2}$, $\left(3 c_{1}\right)^{1 / 3}<\tau<0$, for some constant $c_{1}<0$, the intermediate solution is $u_{M}(t)=\frac{3}{4} t^{2}$, and the righthand solution $u_{R}$ is given parametrically by $t=\frac{2}{3} \tau$ $+c_{2} \tau^{-2}, u_{R}=2 t \tau-\tau^{2}, 0<\tau<\left(3 c_{2}\right)^{1 / 3}$, for some constant $c_{2}>0$. We have that $u_{L}$ intersects $u_{M}$ at $t_{1}$ in $(-1,0)$ and $u_{M}$ intersects $u_{R}$ at $t_{2}$ in $(0,1)$, with $u_{L}^{\prime}\left(t_{1}\right)=t_{1} / 2>u_{M}^{\prime}\left(t_{1}\right)=\frac{3}{2} t_{1}$ and $u_{M}^{\prime}\left(t_{2}\right)=\frac{3}{2} t_{2}>u_{R}^{\prime}\left(t_{2}\right)=t_{2} / 2$. The stability requirements are met since $f_{y^{\prime}}\left[u_{L}(t)\right]=-\frac{2}{3} \tau+2 c_{1} \tau^{-2}>0$,

$$
\begin{gathered}
-1 \leqslant t \leqslant t_{1}, \quad f_{y^{\prime}}\left[u_{M}(t)\right]=t, \quad t_{1} \leqslant t \leqslant t_{2}, \quad \text { and } \\
f_{y^{\prime}}\left[u_{R}(t)\right]=-\frac{2}{3} \tau+2 c_{2} \tau^{-2}<0, \quad t_{2} \leqslant t \leqslant 1 .
\end{gathered}
$$

Finally the crossing conditions hold also, i.e.,

$$
\begin{array}{ll}
f\left(t_{1}, \frac{3}{4} t_{1}^{2}, \omega\right)=\left(\omega-\frac{3}{2} t_{1}\right)\left(\omega-\frac{1}{2} t_{1}\right)<0, & \frac{3}{2} t_{1}<\omega<\frac{1}{2} t_{1}, \\
f\left(t_{2}, \frac{3}{4} t_{2}^{2}, \omega\right)=\left(\omega-\frac{3}{2} t_{2}\right)\left(\omega-\frac{1}{2} t_{2}\right)<0, & \frac{1}{2} t_{2}<\omega<\frac{3}{2} t_{2} .
\end{array}
$$

Since $f_{y} \equiv 1$, we conclude from Theorem 3.2 that this problem has a unique solution $y=y(t, \varepsilon)$ satisfying

$$
\begin{aligned}
& y(t, \varepsilon)=u_{L}(t)+O(\varepsilon), \quad-1 \leqslant t \leqslant t_{1}, \\
& y(t, \varepsilon)=\frac{3}{4} t^{2}+O(\varepsilon), \quad t_{1} \leqslant t \leqslant t_{2}, \\
& y(t, \varepsilon)=u_{R}(t)+O(\varepsilon), \quad t_{2} \leqslant t \leqslant 1 .
\end{aligned}
$$

EXAMPLE 3.4. Finally we illustrate an application of Theorem 3.3. Take the problem

$$
\varepsilon y^{\prime \prime}=y-y^{\prime 3}-t y^{\prime 2}, \quad-2<t<1, \quad y(-2, \varepsilon)=0, \quad y(1, \varepsilon)=2 .
$$

The reduced solutions here are $u_{L}(t) \equiv 0$ and $u_{R}(t)=t+1$ which meet at an angle at $t_{0}=-1$. Clearly $\underline{f}_{y}[0] \equiv 0,-2 \leqslant t \leqslant-1$, and $f_{y}[t+1]=-3-$ 
$2 t<0,-1 \leqslant t \leqslant 1$. The crossing condition $f(-1,0, \omega)=\omega^{2}(1-\omega)>0$, $0<\omega<1$, also holds, and since $f_{y} \equiv 1$, we conclude from Theorem 3.3 that this problem has a unique solution $y=y(t, \varepsilon)$ for which

$$
\begin{aligned}
& y(t, \varepsilon)=O\left(\varepsilon^{1 / 2}\right), \quad-2 \leqslant t \leqslant-1, \quad \text { and } \\
& y(t, \varepsilon)=t+1+O\left(\varepsilon^{1 / 2}\right), \quad-1 \leqslant t \leqslant 1 .
\end{aligned}
$$

4. Generalized $f_{y}$-stability. We consider now the effects of replacing the stability assumptions implied by the positivity of $f_{y}$ with more general but similar conditions. These restrictions have been treated in the papers of Boglaev [1] and the author [6] for the case of problem (3.1), (3.2) with $f_{y^{\prime}} \equiv 0$ or $f_{y}\left[u_{L}, u_{R}\right] \equiv 0$.

For simplicity we shall state the hypotheses regarding generalized $f_{y}$-stability below, and then reformulate Theorems 3.1-3.3 as appropriate. In each of the hypotheses, $f$ is assumed to be sufficiently differentiable with respect to $y$.

H1. There exists a nonnegative integer $q$ and a positive constant $m$ such that

$$
\partial_{y}^{j} f\left(t, u, u^{\prime}\right) \equiv 0, \quad 1 \leqslant j \leqslant 2 q,
$$

for $u=u_{L}, u_{M}$ or $u_{R}$ (the solutions of the reduced equation discussed in $\S 3$ ); in addition,

$$
\partial_{y}^{2 q+1} f\left(t, u, u^{\prime}\right) \geqslant m>0 \text { for such } u .
$$

These relations are to hold on each subinterval where $u_{L}, u_{M}$ or $u_{R}$ defines the reduced solution.

Similar remarks apply to $\mathrm{H} 2$ and $\mathrm{H} 3$ as well.

H2. There exists a positive integer $p$ and a positive constant $m$ such that

$$
\partial_{y}^{j} f\left(t, u, u^{\prime}\right) \geqslant 0, \quad 1 \leqslant j<p,
$$

provided $u^{\prime \prime} \geq 0$; in addition,

$$
\partial_{y}^{p} f\left(t, u, u^{\prime}\right) \geqslant m>0 .
$$

H3. There exists a positive integer $p$ and a positive constant $m$ such that

$$
\begin{array}{ll}
\partial_{y}^{j} f\left(t, u, u^{\prime}\right) \geqslant 0, & 1 \leqslant j<p, j \text { odd, } \\
\partial_{y}^{k} f\left(t, u, u^{\prime}\right) \leqslant 0, & 2 \leqslant k<p, k \text { even, }
\end{array}
$$

provided $u^{\prime \prime}<0$; in addition,

$$
\begin{aligned}
& \partial_{y}^{p} f\left(t, u, u^{\prime}\right) \geqslant m>0, \quad \text { if } p \text { is odd, while } \\
& \partial_{y}^{p} f\left(t, u, u^{\prime}\right) \leqslant-m<0, \quad \text { if } p \text { is even. }
\end{aligned}
$$

THEOREM 4.1(4.2)(4.3). Make the same assumptions as in Theorem 3.1 (3.2)(3.3) with the exceptions that assumption (4) is replaced by $\mathrm{H} 1, \mathrm{H} 2$ or $\mathrm{H} 3$ in any of the theorems, the signs of $u^{\prime \prime}$ are modified accordingly, and the terms of 
order $O(\varepsilon)$ are replaced by terms of order $O\left(\varepsilon^{1 /(2 q+1)}\right), O\left(\varepsilon^{1 / p}\right)$, respectively. Moreover, in the statement of Theorem 3.3, the terms of order $O\left(\varepsilon^{1 / 2}\right)$ are replaced by terms of order $O\left(\varepsilon^{1 /(2 q+2)}\right), O\left(\varepsilon^{1 /(p+1)}\right)$ respectively.

Then the conclusion of Theorem 3.1(3.2)(3.3) is valid with the order terms so modified.

These theorems are proved in a similar fashion as Theorems 3.1-3.3. We expand the term $f\left(t, \sigma, u^{\prime}\right)-f\left(t, u, u^{\prime}\right)(\sigma=\alpha$ or $\beta)$ as follows:

$$
\begin{aligned}
f\left(t, \sigma, u^{\prime}\right)-f\left(t, u, u^{\prime}\right)= & \sum_{j=1}^{r-1} \frac{1}{j !} \partial_{y}^{j} f\left(t, u, u^{\prime}\right)(\sigma-u)^{j} \\
& +\frac{1}{r !} \partial_{y}^{r} f\left(t, u+\theta(\sigma-u), u^{\prime}\right)(\sigma-u)^{r},
\end{aligned}
$$

$0<\theta<1$, and $r=2 q+1$ or $p$, relative to $\mathrm{H} 1, \mathrm{H} 2$ or $\mathrm{H} 3$. Then we proceed as above.

In solving an actual problem it may be necessary to impose various combinations of generalized $f_{y}$-stability on various subintervals of the domain of existence. The adaptation of these theorems to such cases is straightforward.

In conclusion we give one example.

EXAMPLE 4.1. Consider the problem

$$
\begin{aligned}
\varepsilon y^{\prime \prime} & =y^{2}-t^{2} y^{\prime 2}, \quad-1<t<1 \\
y(-1, \varepsilon) & =A, \quad y(1, \varepsilon)=B, \quad-B<A<0 .
\end{aligned}
$$

The stable solutions of the reduced equation are $u_{L}(t)=-A t^{-1},-1 \leqslant t<$ 0 , and $u_{R}(t)=B t,-1 \leqslant t \leqslant 1$, since $f_{y}\left[u_{L}(t)\right] \equiv-2 A>0$ and $f_{y}\left[u_{R}(t)\right]=$ $-2 B t^{2}$. Clearly, $u_{L}\left(t_{0}\right)=u_{R}\left(t_{0}\right)$ and $u_{L}^{\prime}\left(t_{0}\right)=-B<u_{R}^{\prime}\left(t_{0}\right)=B$, for $t_{0}=$ $-\left(-A B^{-1}\right)^{1 / 2}$ in $(-1,0)$. In addition, $f_{y}[B t]=2 B t>0,0 \leqslant t<1$, and $f_{y y} \equiv 2$. Although $f_{y}[B t]<0, t_{0}<t<0$, there is no real difficulty because $u_{R}^{\prime \prime} \equiv 0$.

Finally the crossing condition at $t_{0}$ is satisfied. It follows from Theorem 4.1 that this problem has a solution $y=y(t, \varepsilon)$ for which

$$
u(t)-\varepsilon^{1 / 2} \gamma \leqslant y(t, \varepsilon)<u(t), \quad-1 \leqslant t \leqslant 1,
$$

where

$$
u(t)= \begin{cases}-A t^{-1}, & -1 \leqslant t \leqslant t_{0}, \\ B t, & t_{0} \leqslant t \leqslant 1 .\end{cases}
$$

5. Singular Haber-Levinson crossings. We consider now the situation in which both of the reduced solutions $u_{L}, u_{R}$ annihilate the partial derivative $f_{y^{\prime}}$ in a neighborhood of the point of intersection $t_{0}{ }^{7}$ The term "singular" is

${ }^{7}$ That is, $f_{y^{\prime}}\left(t, u_{L}(t), u_{L}^{\prime}(t)\right)=f_{y^{\prime}}\left(t, u_{R}(t), u_{R}^{\prime}(t)\right) \equiv 0$. 
applied to such a crossing since in this neighborhood a solution behaves like a classical singular solution of a first-order differential equation. We focus attention on a class of equations for which $f\left(t_{0}, u_{L}\left(t_{0}\right), \omega\right) \equiv 0, \omega$ in $\left(u_{L}^{\prime}\left(t_{0}\right)\right.$, $\left.u_{R}^{\prime}\left(t_{0}\right)\right)$ or $\left(u_{R}^{\prime}\left(t_{0}\right), u_{L}^{\prime}\left(t_{0}\right)\right)$. Since Theorem 2.1 is inapplicable to problem (3.1), (3.2) with this "vanishing" property, we must appeal to Theorem 2.2 and therefore assume $f=O\left(\left|y^{\prime}\right|^{2}\right)$, as $\left|y^{\prime}\right| \rightarrow \infty$.

THEOREM 5.1. Assume

(1) the reduced equation $f\left(t, u, u^{\prime}\right)=0$ has two solutions $u=u_{L}(t), u=$ $u_{R}(t)$, defined and of class $C^{(2)}$ on $\left[a, t_{0}\right],\left[t_{0}, b\right]$, respectively, $a<t_{0}<b$, with $u_{L}(a)=A, u_{L}\left(t_{0}\right)=u_{R}\left(t_{0}\right), u_{L}^{\prime}\left(t_{0}\right) \neq u_{R}^{\prime}\left(t_{0}\right)$, and $u_{R}(b)=B$;

(2) the function $f$ is continuous in all of its arguments and of class $C^{(1)}$ with respect to $y$ and $y^{\prime}$ in $\mathcal{E}: a \leqslant t \leqslant b, y-u(t)=O\left(\varepsilon^{1 / 2}\right), y^{\prime}-u^{\prime}(t)=$ $O(d(t, \varepsilon))$, where

$$
u(t)= \begin{cases}u_{L}(t), & a \leqslant t \leqslant t_{0}, \\ u_{R}(t), & t_{0} \leqslant t \leqslant b,\end{cases}
$$

and $d(t, \varepsilon)=O\left(\varepsilon^{1 / 2}\right)$, for $t$ in $[a, b] \backslash\left(t_{0}-\delta, t_{0}+\delta\right), d(t, \varepsilon)=O\left(\mid u_{L}^{\prime}\left(t_{0}\right)-\right.$ $\left.u_{R}^{\prime}\left(t_{0}\right) \mid\right)$, for $t$ in $\left(t_{0}-\delta, t_{0}+\delta\right)$; here $\delta$ is a small positive constant; in addition, $f=O\left(\left|y^{\prime}\right|^{2}\right)$, as $\left|y^{\prime}\right| \rightarrow \infty$;

(3) for $t$ in $\left[a, t_{0}\right], f_{y^{\prime}}\left(t, u_{L}(t), \xi_{1}(t)\right) \geqslant 0$, and for $t$ in $\left[t_{0}, b\right], f_{y^{\prime}}\left(t, u_{R}(t)\right.$, $\left.\xi_{2}(t)\right) \leqslant 0$, where $\xi_{1}(t)=u_{L}^{\prime}(t)+O(d(t, \varepsilon))$ and $\xi_{2}(t)=u_{R}^{\prime}(t)+O(d(t, \varepsilon))$;

(4) there exists a positive constant $l$ such that

$$
\begin{array}{ll}
f_{y}\left(t, u_{L}(t), u_{L}^{\prime}(t)\right) \geqslant l>0, & a \leqslant t \leqslant t_{0}, \quad \text { and } \\
f_{y}\left(t, u_{R}(t), u_{R}^{\prime}(t)\right) \geqslant l>0, & t_{0} \leqslant t \leqslant b .
\end{array}
$$

Then there exists an $\varepsilon_{0}>0$ such that for each $\varepsilon, 0<\varepsilon \leqslant \varepsilon_{0}$, the problem (3.1), (3.2) has a solution $y=y(t, \varepsilon)$. Moreover, for

$$
\begin{gathered}
u(t)=\left\{\begin{array}{l}
u_{L}(t), \quad a \leqslant t \leqslant t_{0}, \\
u_{R}(t), \quad t_{0} \leqslant t \leqslant b,
\end{array}\right. \\
y(t, \varepsilon)=u(t)+O\left(\varepsilon^{1 / 2}\right), \quad a \leqslant t \leqslant b .
\end{gathered}
$$

Proof. The proof reduces to constructing suitable bounding functions $\alpha, \beta$ which satisfy the hypotheses of Theorem 2.2. Suppose, for definiteness, that $u_{L}^{\prime}\left(t_{0}\right)<u_{R}^{\prime}\left(t_{0}\right)$, and define for $\varepsilon>0$,

$$
\alpha(t, \varepsilon)= \begin{cases}u_{L}(t)-\varepsilon \gamma l^{-1}, & a \leqslant t \leqslant t_{0}, \\ u_{R}(t)-\varepsilon \gamma 1^{-1}, & t_{0} \leqslant t \leqslant b,\end{cases}
$$

and 
$\beta(t, \varepsilon)=\left\{\begin{array}{l}u_{L}(t)+\varepsilon^{1 / 2} \gamma_{1} l^{-1}, a \leqslant t \leqslant t_{0}, \\ u_{R}(t)+\left(\varepsilon l^{-1}\right)^{1 / 2}\left(u_{R}^{\prime}\left(t_{0}\right)-u_{L}^{\prime}\left(t_{0}\right)\right) \exp \left[\begin{array}{l}\left.-\left(\varepsilon l^{-1}\right)^{-1 / 2}\left(t-t_{0}\right)\right] \\ +\varepsilon \gamma l^{-1}, \quad t_{0} \leqslant t \leqslant b .\end{array}\right.\end{array}\right.$

Clearly, $\alpha \leqslant \beta, \alpha(a, \varepsilon) \leqslant A \leqslant \beta(a, \varepsilon)$ and $\alpha(b, \varepsilon) \leqslant B \leqslant \beta(b, \varepsilon)$. The verification that $\alpha, \beta$ satisfy the correct differential inequalities is straightforward and is omitted.

We remark that assumption (3) places an additional restriction on $u_{L}$ and $u_{R}$ in that near $t=t_{0}$ not only must $f_{y^{\prime}}\left(t, u_{L}(t), u_{L}^{\prime}(t)\right)$ and $f_{y^{\prime}}\left(t, u_{L}(t), u_{R}^{\prime}(t)\right)$ vanish identically but also $f_{y^{\prime}}\left(t, u_{L}(t), \xi(t)\right)$ must be nonnegative and $f_{y^{\prime}}\left(t, u_{R}(t), \xi(t)\right)$ must be nonpositive for all $\xi(t)$ between $u_{L}^{\prime}(t)$ and $u_{R}^{\prime}(t)$. We note finally that this theorem may be extended to cover cases involving multiple crossings, as well as to cases where more general $y$-stability assumptions are made. Three examples are now given.

EXAMPLE 5.1. Consider the problem

$$
\begin{gathered}
\varepsilon y^{\prime \prime}=(y-t) y^{\prime}-t y^{2}+y, \quad-1<t<1, \\
y(-1, \varepsilon)=-1, \quad y(1, \varepsilon)=0 .
\end{gathered}
$$

The stable solutions of the reduced equation are $u_{L}(t)=t$ and $u_{R}(t) \equiv 0$. Here $f_{y^{\prime}}\left(t, u_{L}(t), \xi\right)=-2 t \xi \geqslant 0,-\delta \leqslant t \leqslant 0, u_{R}^{\prime}(0)=0<\xi<u_{L}^{\prime}(0)=1$; $f_{y^{\prime}}\left(t, u_{R}(t), \xi\right)=-2 t \xi \leqslant 0,0 \leqslant t \leqslant \delta, 0<\xi<1$. Since $f_{y}\left[u_{L}(t)\right] \equiv 2$ and $f_{y}\left[u_{R}(t)\right] \equiv 1$, we deduce from Theorem 5.1 the existence of a solution $y=y(t, \varepsilon)$ satisfying

$$
\min \{t, 0\}-\varepsilon^{1 / 2} \gamma \leqslant y(t, \varepsilon) \leqslant \min \{t, 0\}, \quad-1 \leqslant t \leqslant 1^{8}
$$

EXAMPLE 5.2. In this example we observe a regular-singular crossing, i.e., the intersection of a singular solution with a semistable one. Consider

$$
\begin{aligned}
\varepsilon y^{\prime \prime} & =(y-t) y^{\prime}, \quad 0<t<1, \\
y(0, \varepsilon) & =0, \quad y(1, \varepsilon)=B, \quad 0<B<1 .
\end{aligned}
$$

Of the reduced solutions, $u_{L}(t)=t$ and $u_{R}(t) \equiv B$ are the pair of interest, with $f_{y^{\prime}}\left(t, u_{L}(t), \xi\right) \equiv 0$, and $f_{y^{\prime}}\left(t, u_{R}(t), \xi\right)=B-t \leqslant 0, B \leqslant t \leqslant 1$ and $0<\xi$ $<1$. Thus $u_{L}(t)$ is singular, but stable since $f_{y}\left[u_{L}(t)\right] \equiv 1$. On the other hand, $f_{y}\left[u_{R}(t)\right] \equiv 0$, so Theorem 5.1 is not immediately applicable. Still one may argue as above, since $u_{R}^{\prime \prime} \equiv 0$, to deduce the existence of a solution $y=y(t, \varepsilon)$ satisfying

$$
\min \{t, B\}-\varepsilon^{1 / 2} \gamma \leqslant y(t, \varepsilon) \leqslant \min \{t, B\}, \quad 0 \leqslant t \leqslant 1 .{ }^{9}
$$

EXAMPLE 5.3. We consider finally the case of a singular-singular crossing,

${ }^{8}$ There is no term of order $O\left(\varepsilon^{1 / 2}\right)$ on the right side of the inequality because $u_{L}^{\prime \prime}=u_{R}^{\prime \prime} \equiv 0$.

${ }^{9}$ See footnote 8. 
i.e., the intersection of two singular solutions. The problem is

$$
\begin{gathered}
\varepsilon y^{\prime \prime}=y y^{\prime 2}-2 t y^{\prime}+y, \quad-1<t<1, \\
y(-1, \varepsilon)=1, \quad y(1, \varepsilon)=1 .
\end{gathered}
$$

The reduced solutions are the singular ones $u_{L}(t)=-t,-1 \leqslant t \leqslant 0$, and $u_{R}(t)=t, 0 \leqslant t \leqslant 1$, which are stable since $f_{y}[|t|] \equiv 2$. Moreover,

$$
\begin{aligned}
& f_{y^{\prime}}\left(t, u_{L}(t), \xi\right)=-2 t(\xi+1) \geqslant 0, \quad-\delta \leqslant t \leqslant 0,-1<\xi<1 ; \\
& f_{y^{\prime}}\left(t, u_{R}(t), \xi\right)=2 t(\xi-1)<0, \quad 0<t \leqslant \delta,-1<\xi<1 .
\end{aligned}
$$

Thus we can apply Theorem 5.1 to deduce the existence of a unique solution $y=y(t, \varepsilon)$ satisfying

$$
|t| \leqslant y(t, \varepsilon) \leqslant|t|+\varepsilon^{1 / 2} \gamma, \quad-1 \leqslant t \leqslant 1 .
$$

6. Boundary layer behavior. We recall that in the formulation of Haber and Levinson [4] as well as in the previous three sections the assumption is made that the solutions of the reduced equation satisfy a boundary condition at the respective endpoint. Such a restriction naturally precludes the occurrence of boundary layer behavior, i.e., nonuniform convergence of the solution $y(t, \varepsilon)$ as $\varepsilon \rightarrow 0^{+}$for $t$ near $a$ or $b$. Indeed, the nonuniformity in the solution first manifests itself in the derivative $y^{\prime}(t, \varepsilon)$ and it is localized to the point(s) of the angular crossing of the reduced solutions. If, however, one modifies the original assumptions in [4] by considering various forms of stability and if one, for example, studies singular reduced solutions, then it is reasonable to expect the occurrence of boundary layer behavior. ${ }^{10}$ The essential restriction is that in (3.1) $f\left(t, y, y^{\prime}\right)=O\left(\left|y^{\prime}\right|^{2}\right)$, as $\left|y^{\prime}\right| \rightarrow \infty$. Such a growth restriction is a necessary condition for boundary layer behavior; see, e.g., [14] and the later discussion in [12, Chapter 2]. The three theorems presented below are basically extensions of Theorem 5.1 to include the presence of boundary layers.

THEOREM 6.1. Assume

(1) the reduced equation $f\left(t, u, u^{\prime}\right)=0$ has two solutions $u=u_{L}(t)$ and $u=u_{S}(t)$, defined and of class $C^{(2)}$ on $\left[a, t_{0}\right]$ and $\left[t_{0}, b\right]$, respectively, $a<t_{0}<$ $b$, with $u_{L}(a)=A, u_{L}\left(t_{0}\right)=u_{S}\left(t_{0}\right), u_{L}^{\prime}\left(t_{0}\right) \neq u_{S}^{\prime}\left(t_{0}\right)$, and $u_{S}(b) \neq B$;

(2) the function $f$ is continuous in all of its arguments and of class $C^{(2)}$ with respect to $y$ and $y^{\prime}$ in $\sigma: a \leqslant t \leqslant b, y-u(t)=O\left(d_{1}(t, \varepsilon)\right), y^{\prime}-u^{\prime}(t)=$ $O\left(d_{2}(t, \varepsilon)\right), a \leqslant t \leqslant b-\delta,\left|y^{\prime}\right|<\infty, b-\delta<t \leqslant b$, where

$$
u(t)= \begin{cases}u_{L}(t), & a \leqslant t \leqslant t_{0}, \\ u_{S}(t), & t_{0} \leqslant t \leqslant b,\end{cases}
$$

$\delta$ is a small positive constant, $d_{1}(t, \varepsilon)=O\left(\varepsilon^{1 / 2}\right), a \leqslant t \leqslant b-\delta, d_{1}(t, \varepsilon)=$

\footnotetext{
${ }^{10}$ This is because singular solutions $u_{S}$ satisfy $f_{y}\left[u_{S}\right] \equiv 0$ and so they cannot in general be made to satisfy either of the original boundary conditions.
} 
$O\left(\left|u_{S}(b)-B\right|\right), b-\delta<t \leqslant b$, and $d_{2}(t, \varepsilon)=O\left(\varepsilon^{1 / 2}\right),\left\{a<t<t_{0}-\delta\right\} \cup$ $\left\{t_{0}+\delta \leqslant t \leqslant b-\delta\right\}, d_{2}(t, \varepsilon)=O\left(\left|u_{L}^{\prime}\left(t_{0}\right)-u_{s}^{\prime}\left(t_{0}\right)\right|\right), t_{0}-\delta<t<t_{0}+\delta$; moreover, $f_{y^{\prime} y^{\prime}}=O(1)$, as $\left|y^{\prime}\right| \rightarrow \infty$, for $\left(t, y, y^{\prime}\right)$ in $\mathrm{T}$;

(3) for $t$ in $\left[a, t_{0}\right], f_{y^{\prime}}\left(t, u_{L}(t), u_{L}^{\prime}(t)\right) \geqslant 0$; while $f_{y^{\prime}}\left(t, u_{S}(t), \xi\right) \leqslant 0, t_{0} \leqslant t \leqslant t_{0}$ $+\delta$, $\xi$ in $\left(u_{L}^{\prime}\left(t_{0}\right), u_{S}^{\prime}\left(t_{0}\right)\right)$ or $\left(u_{S}^{\prime}\left(t_{0}\right), u_{L}^{\prime}\left(t_{0}\right)\right)$, and $f_{y^{\prime}}\left(t, \eta, u_{S}^{\prime}(t)\right) \geqslant 0, b-\delta<t$ $<b, \eta-u_{S}(t)=O\left(d_{1}(t, \varepsilon)\right)$

(4) there exists a positive constant $l$ such that

$$
\begin{gathered}
f_{y}\left(t, u_{L}(t), u_{L}^{\prime}(t)\right) \geqslant l>0, \quad a \leqslant t \leqslant t_{0}, \quad \text { and } \\
f_{y}\left(t, \eta, u_{S}^{\prime}(t)\right) \geqslant l>0, \quad t_{0} \leqslant t \leqslant b, \eta-u_{S}(t)=O\left(d_{1}\right) ;
\end{gathered}
$$

(5) there exists a positive constant $p$ such that

$$
\left(B-u_{S}(b)\right) f_{y^{\prime} y^{\prime}} \geqslant p>0 \text { in } \mathcal{T} \cap[b-\delta, b] .
$$

Then there exists an $\varepsilon_{0}>0$ such that for each $\varepsilon, 0<\varepsilon \leqslant \varepsilon_{0}$, the problem (3.1), (3.2) has a solution $y=y(t, \varepsilon)$. In addition,

$$
\begin{gathered}
y(t, \varepsilon)=u_{L}(t)+O\left(\varepsilon^{1 / 2}\right), \quad a \leqslant t \leqslant t_{0}, \\
y(t, \varepsilon)=u_{S}(t)+O\left(\left|B-u_{S}(b)\right| \exp \left[-\left(\varepsilon^{-1} l\right)^{1 / 2}(b-t)\right]\right)+O\left(\varepsilon^{1 / 2}\right), \\
t_{0} \leqslant t \leqslant b .
\end{gathered}
$$

THEOREM 6.2. Assume

(1) the reduced equation $f\left(t, u, u^{\prime}\right)=0$ has two solutions $u=u_{1}(t)$ and $u=u_{2}(t)$, defined and of class $C^{(2)}$ on $\left[a, t_{0}\right]$ and $\left[t_{0}, b\right]$, respectively, with $u_{1}(a) \neq A, u_{1}\left(t_{0}\right)=u_{2}\left(t_{0}\right), u_{1}^{\prime}\left(t_{0}\right) \neq u_{2}^{\prime}\left(t_{0}\right)$, and $u_{2}(b) \neq B$

(2) the function $f$ is continuous in all arguments and of class $C^{(2)}$ with respect to $y$ and $y^{\prime}$ in $\mathcal{G}: a \leqslant t \leqslant b, y-u(t)=O\left(d_{1}(t, \varepsilon)\right), y^{\prime}-u^{\prime}(t)=O\left(d_{2}(t, \varepsilon)\right)$, $a+\delta \leqslant t \leqslant b-\delta,\left|y^{\prime}\right|<\infty,\{a \leqslant t \leqslant a+\delta\} \cup\{b-\delta \leqslant t \leqslant b\}$, where

$$
u(t)= \begin{cases}u_{1}(t), & a \leqslant t \leqslant t_{0}, \\ u_{2}(t), & t_{0} \leqslant t \leqslant b,\end{cases}
$$

$\delta$ is a small positive constant, $d_{1}(t, \varepsilon)=O\left(\left|u_{1}(a)-A\right|\right), a \leqslant t<a+\delta$, $d_{1}(t, \varepsilon)=O\left(\varepsilon^{1 / 2}\right), a+\delta \leqslant t \leqslant b-\delta, d_{1}(t, \varepsilon)=O\left(\left|u_{2}(b)-B\right|\right), b-\delta<t$ $\leqslant b$, and $d_{2}(t, \varepsilon)=O\left(\varepsilon^{1 / 2}\right),\left\{a+\delta \leqslant t \leqslant t_{0}-\delta\right\} \cup\left\{t_{0}+\delta \leqslant t \leqslant b-\delta\right\}$, $d_{2}(t, \varepsilon)=O\left(\left|u_{1}^{\prime}\left(t_{0}\right)-u_{2}^{\prime}\left(t_{0}\right)\right|\right), t_{0}-\delta<t<t_{0}+\delta$; moreover, $f_{y^{\prime} y^{\prime}}=O(1)$, as $\left|y^{\prime}\right| \rightarrow \infty$ in $\mathcal{G}$;

(3) for $t$ in $[a, a+\delta), f_{y^{\prime}}\left(t, \eta_{1}, u_{1}^{\prime}(t)\right) \leqslant 0, \eta_{1}-u_{1}(t)=O\left(d_{1}(t, \varepsilon)\right)$; for $t$ in $\left(t_{0}-\delta, t_{0}\right], f_{y^{\prime}}\left(t, u_{1}(t), \xi\right) \geqslant 0, \xi$ in $\left(u_{1}^{\prime}\left(t_{0}\right), u_{2}^{\prime}\left(t_{0}\right)\right)$ or $\left(u_{2}^{\prime}\left(t_{0}\right), u_{1}^{\prime}\left(t_{0}\right)\right)$; while for $t$ in $\left[t_{0}, t_{0}+\delta\right), f_{y^{\prime}}\left(t, u_{2}(t), \xi\right) \leqslant 0$, same $\xi$ as above, and for $t$ in $(b-\delta, b]$, $f_{y^{\prime}}\left(t, \eta_{2}, u_{2}^{\prime}\right) \geqslant 0, \eta_{2}-u_{2}(t)=O\left(d_{1}(t, \varepsilon)\right)$

(4) there exists a positive constant $l$ such that 


$$
\begin{array}{ll}
f_{y}\left(t, \eta_{1}, u_{1}^{\prime}(t)\right) \geqslant l>0, & a \leqslant t \leqslant t_{0}, \eta_{1}-u_{1}(t)=O\left(d_{1}(t, \varepsilon)\right), \quad \text { and } \\
f_{y}\left(t, \eta_{2}, u_{2}^{\prime}(t)\right) \geqslant l>0, & t_{0} \leqslant t \leqslant b, \eta_{2}-u_{2}(t)=O\left(d_{1}(t, \varepsilon)\right) ;
\end{array}
$$

(5) there exists a positive constant $p$ such that

$$
\begin{aligned}
& \left(A-u_{1}(a)\right) f_{y^{\prime} y^{\prime}} \geqslant p>0, \quad \text { in } \mathcal{S} \cap[a, a+\delta), \quad \text { and } \\
& \left(B-u_{2}(b)\right) f_{y^{\prime} y^{\prime}} \geqslant p>0, \quad \text { in } \mathcal{S} \cap(b-\delta, b] .
\end{aligned}
$$

Then there exists an $\varepsilon_{0}>0$ such that for each $\varepsilon, 0<\varepsilon \leqslant \varepsilon_{0}$, the problem (3.1), (3.2) has a solution $y=y(t, \varepsilon)$. In addition, for

$$
\begin{gathered}
u(t)= \begin{cases}u_{1}(t), & a \leqslant t \leqslant t_{0}, \\
u_{2}(t), & t_{0} \leqslant t \leqslant b,\end{cases} \\
y(t, \varepsilon)=u(t)+O\left(\left|A-u_{1}(a)\right| \exp \left[-\left(\varepsilon^{-1} l\right)^{1 / 2}(t-a)\right]\right) \\
+O\left(\left|B-u_{2}(b)\right| \exp \left[-\left(\varepsilon^{-1} l\right)^{1 / 2}(b-t)\right]\right)+O\left(\varepsilon^{1 / 2}\right), \quad a \leqslant t \leqslant b .
\end{gathered}
$$

To prove these two theorems we introduce into the bounding functions $\alpha, \beta$ given in $\$ 5$ the boundary layer terms $\left|A-u_{1}(a)\right| \exp \left[-\left(\varepsilon^{-1} l\right)^{1 / 2}(t-a)\right]$ and $\left|B-u_{2}(b)\right| \exp \left[-\left(\varepsilon^{-1} l\right)^{1 / 2}(b-t)\right]$. The functions $f[\alpha]$ and $f[\beta]$ are here expanded to quadratic terms in $y^{\prime}$. As an illustration, suppose we consider just $\left[a, t_{0}\right]$ and let $u_{1}(a)>A$. Then we define the function

$$
\alpha(t, \varepsilon)=u_{1}(t)-\left(u_{1}(a)-A\right) E(t, \varepsilon)-\varepsilon \gamma l^{-1},
$$

where $E(t, \varepsilon)=\exp \left[-\left(\varepsilon^{-1} l\right)^{1 / 2}(t-a)\right]$. Differentiating and substituting into $\varepsilon \alpha^{\prime \prime}-f[\alpha]$ we have

$$
\begin{aligned}
\varepsilon \alpha^{\prime \prime}-f\left(t, \alpha, \alpha^{\prime}\right)= & \varepsilon u_{1}^{\prime \prime}-l\left(u_{1}(a)-A\right) E(t, \varepsilon) \\
& -f\left[u_{1}\right]-f_{y}[t, \varepsilon]\left\{-\left(u_{1}(a)-A\right) E(t, \varepsilon)-\varepsilon \gamma l^{-1}\right\} \\
& -f_{y^{\prime}}\left(t, \alpha, u_{1}^{\prime}\right)\left\{\left(\varepsilon^{-1} l\right)^{1 / 2}\left(u_{1}(a)-A\right) E(t, \varepsilon)\right\} \\
& -\frac{1}{2} f_{y^{\prime} y^{\prime}}\{t, \varepsilon\}\left\{\left(\varepsilon^{-1} l\right)^{1 / 2}\left(u_{1}(a)-A\right) E(t, \varepsilon)\right\}^{2} .
\end{aligned}
$$

for $[t, \varepsilon]=\left(t, u_{1} \check{+} \theta_{1}\left(\alpha-u_{1}\right), u_{1}^{\prime}\right)$ and $\{t, \varepsilon\}=\left(t, \alpha, u_{1}^{\prime}+\theta_{2}\left(\alpha^{\prime}-u_{1}^{\prime}\right)\right), 0<$ $\theta_{1}, \theta_{2}<1$. Consider first $[a, a+\delta)$, then

$$
\begin{aligned}
\varepsilon \alpha^{\prime \prime}-f\left(t, \alpha, \alpha^{\prime}\right) \geqslant & -\varepsilon M \pm l\left(u_{1}(a)-A\right) E(t, \varepsilon) \\
& +\varepsilon \gamma+\frac{1}{2} p \varepsilon^{-1} l\left(u_{1}(a)-A\right)^{2} E^{2}(t, \varepsilon) \\
\geqslant & 0, \quad \text { if } \gamma \geqslant M \quad\left(\left|u_{1}^{\prime \prime}\right| \leqslant M\right) .
\end{aligned}
$$

On $\left(a+\delta, t_{0}\right]$, the function $E(t, \varepsilon)$ is transcendentally small. Consequently, if $\gamma=M+1$, then for $\varepsilon$ sufficiently small, we again have the required inequality. The sign restriction on $f_{y^{\prime} y^{\prime}}$ near $t=a$ is necessary for the above method of proof to succeed; however, it has a natural geometric 
interpretation. Since inside of a boundary layer, the derivative of a solution $y(t, \varepsilon)$ is unbounded as a function of $\varepsilon$, the curvature of $y$ is determined by the sign of $f_{y^{\prime} y^{\prime}}$, i.e., $y^{\prime \prime} \cong \frac{1}{2} \varepsilon^{-1} f_{y^{\prime} y^{\prime}} y^{\prime 2}$. For example, if $f_{y^{\prime} y^{\prime}}>0$ near $t=a$, then $y$ is convex near $t=a$, and since $u_{1}$ approximates $y$ to the edge of the boundary layer, $u_{1}(a)$ must be less than $A$, i.e., $\left(u_{1}(a)-A\right) f_{y^{\prime} y^{\prime}}<0$.

In the next theorem we consider the situation in which two regular solutions of the reduced equation have a Haber-Levinson crossing in the usual sense, and the solution of the full problem exhibits boundary layer behavior at one or both endpoints. For ease of exposition we consider the case when there is a single boundary layer located at $t=a$.

THEOREM 6.3. Assume

(1) the reduced equation $f\left(t, u, u^{\prime}\right)=0$ has two solutions $u=u_{L}(t), u=$ $u_{R}(t)$, defined and of class $C^{(2)}$ on $\left[a, t_{0}\right],\left[t_{0}, b\right]$, respectively, with $u_{L}(a) \neq A$, $u_{L}\left(t_{0}\right)=u_{R}\left(t_{0}\right), u_{L}^{\prime}\left(t_{0}\right) \neq u_{R}^{\prime}\left(t_{0}\right)$, and $u_{R}(b)=B$;

(2) the function $f$ is continuous in all arguments and of class $C^{(2)}$ with respect to $y$ and $y^{\prime}$ in $\mathcal{H}: a \leqslant t \leqslant b, y-u(t)=O\left(d_{1}(t, \varepsilon)\right),\left|y^{\prime}\right|<\infty, a \leqslant t<a+$ $\delta, y^{\prime}-u^{\prime}(t)=O\left(d_{2}(t, \varepsilon)\right), a+\delta \leqslant t \leqslant b$, where

$$
u(t)= \begin{cases}u_{L}(t), & a \leqslant t \leqslant t_{0}, \\ u_{R}(t), & t_{0} \leqslant t \leqslant b,\end{cases}
$$

$d_{1}(t, \varepsilon)=O\left(\left|u_{L}(a)-A\right|\right), a \leqslant t<a+\delta, d_{1}(t, \varepsilon)=O(\varepsilon), a+\delta \leqslant t \leqslant b$, $d_{2}(t, \varepsilon)=O(\varepsilon),\left\{a+\delta \leqslant t \leqslant t_{0}-\delta\right\} \cup\left\{t_{0}+\delta \leqslant t \leqslant b\right\}$, and $d_{2}(t, \varepsilon)=$ $O\left(\left|u_{L}^{\prime}\left(t_{0}\right)-u_{R}^{\prime}\left(t_{0}\right)\right|\right), t_{0}-\delta<t<t_{0}+\delta$; here $\delta>0$ is a small constant; in addition, $f_{y^{\prime} y^{\prime}}=O(1)$ in $\mathcal{H}$ as $\left|y^{\prime}\right| \rightarrow \infty$;

(3) there exist positive constants $k_{1}, k_{2}$ and $p$ such that $f_{y^{\prime}}\left(t, u_{L}(t), u_{L}^{\prime}(t)\right) \leqslant$ $-k_{1}<0, a \leqslant t<a+\delta ; f_{y^{\prime}}\left(t, u_{L}(t), u_{L}^{\prime}(t)\right) \geqslant k_{2}>0, t_{0}-\delta<t \leqslant t_{0} ;$ $f_{y^{\prime}}\left(t, u_{R}(t), u_{R}^{\prime}(t)\right) \leqslant-k_{2}<0, t_{0} \leqslant t \leqslant b$; and $\left(A-u_{L}(a)\right) f_{y^{\prime} y^{\prime}} \geqslant p>0$, in $\mathcal{H} \cap[a, a+\delta]$

(4) there exists a positive constant $l$ such that

$$
\begin{gathered}
f_{y}\left(t, \eta, u_{L}^{\prime}(t)\right) \geqslant l>0, \quad a \leqslant t \leqslant t_{0}-\delta, \eta-u_{L}(t)=O\left(d_{1}(t, \varepsilon)\right) ; \\
f\left(t_{0}, u_{i}\left(t_{0}\right), \omega\right) \begin{cases}>0, & u_{L}^{\prime}\left(t_{0}\right)<\omega<u_{R}^{\prime}\left(t_{0}\right), \\
<0, & u_{R}^{\prime}\left(t_{0}\right)<\omega<u_{L}^{\prime}\left(t_{0}\right) .\end{cases}
\end{gathered}
$$

Then there exists an $\varepsilon_{0}>0$ such that for each $\varepsilon, 0<\varepsilon \leqslant \varepsilon_{0}$, the problem (3.1), (3.2) has a solution $y=y(t, \varepsilon)$. Moreover, for

$$
\begin{gathered}
u(t)= \begin{cases}u_{L}(t), & a \leqslant t \leqslant t_{0}, \\
u_{R}(t), & t_{0} \leqslant t \leqslant b,\end{cases} \\
y(t, \varepsilon)=u(t)+o\left(\mid A-u_{L}(a) \exp \left[-\bar{k}_{1}(t-a) \varepsilon^{-1}\right]\right)+O(\varepsilon),
\end{gathered}
$$

$a \leqslant t \leqslant b$ for $0<\bar{k}_{1}<k_{1}$. 
Theorem 6.3 is proved by adding the appropriate boundary layer term to the bounding functions defined in the proof of Theorem 3.1.

In conclusion we give some examples.

EXAMPLE 6.1. Consider the problem

$$
\begin{gathered}
\varepsilon y^{\prime \prime}=y-t y^{\prime 2}, \quad-1<t<1, \\
y(-1, \varepsilon)=-1, \quad y(1, \varepsilon)=B<0 .
\end{gathered}
$$

We note that $u_{L}(t)=t\left(u_{S}(t) \equiv 0\right)$ satisfies the differential equation and $u_{L}(-1)=y(-1, \varepsilon)\left(u_{S}(1)>y(1, \varepsilon)\right)$. Consequently the function $\varphi(t)=$ $\min \{t, 0\}$ is an upper solution of this problem, ${ }^{11}$ and we anticipate that the reduced solutions $u_{L}$ and $u_{S}$ will be important in describing the behavior of solutions for small $\varepsilon>0$. Clearly $f_{y}\left(t, u_{L}(t), \xi\right)=-2 t \xi \geqslant 0,-\delta<t<0$, $u_{S}^{\prime}(0)=0<\xi<u_{L}^{\prime}(0)=1$, and $f_{y^{\prime}}\left(t, \eta, u_{S}^{\prime}(t)\right) \equiv 0$. Since $f_{y} \equiv 1, u_{S}(1)>B$, and $f_{y^{\prime} y^{\prime}}=-2 t<0,1-\delta<t<1$, we can apply Theorem 6.1 to deduce the existence of a unique solution $y=y(t, \varepsilon)$ satisfying

$\min \{t, 0\}+B \exp \left[-\varepsilon^{-1 / 2}(1-t)\right]-\varepsilon^{1 / 2} \leqslant y(t, \varepsilon) \leqslant \min \{t, 0\}$,

$-1<t<1$.

EXAMPLE 6.2. We consider next the occurrence of a singular-singular crossing with boundar- layer behavior at each endpoint. The problem is

$$
\begin{array}{rlrl}
\varepsilon y^{\prime \prime} & =|t| y^{\prime 2}-2 t y^{\prime}+y, & -1<t<1, \\
y(-1, \varepsilon) & =A, \quad y(1, \varepsilon)=B, \quad A, B>1 .
\end{array}
$$

The reduced pair consists of the singular solutions $u_{1}(t)=-t,-1<t<0$, $u_{2}(t)=t, 0<t<1$. These functions are stable since $f_{y} \equiv 1 ; 1^{12}$ moreover $u_{1}(-1)<A, u_{2}(1)<B$, and $f_{y^{\prime} y^{\prime}}=2|t|>0$, for $t$ in $[-1,-1+\delta) \cup(1-$ $\delta, 1]$. By Theorem 6.2 there exists a unique solution $y=y(t, \varepsilon)$ such that

$$
\begin{aligned}
|t|<y(t, \varepsilon)< & |t|+(A-1) \exp \left[-\varepsilon^{-1 / 2}(1+t)\right] \\
& +(B-1) \exp \left[-\varepsilon^{-1 / 2}(1-t)\right]+\varepsilon^{1 / 2} \gamma, \quad-1 \leqslant t \leqslant 1 .
\end{aligned}
$$

EXAMPLE 6.3. This example consists of a whole family of problems. Consider

$$
\begin{gathered}
\varepsilon y^{\prime \prime}=h(t, y)\left(1+y^{\prime 2}\right)=H\left(t, y, y^{\prime}\right), \quad-1<t<1, \\
y(-1, \varepsilon)=A, \quad y(1, \varepsilon)=B .
\end{gathered}
$$

Since $1+y^{\prime 2} \geqslant 1$ the existence and the asymptotic behavior of its solutions are determined by the solutions $u=u(t)$ of $h(t, u)=0$. Such solutions are singular because $H_{y^{\prime}}\left(t, u, u^{\prime}\right) \equiv 0$. If, however, two of them $u_{1}$ and $u_{2}$ intersect at a point $t_{0}$ in $(-1,1)$ then the full $(\varepsilon>0)$ problem can be shown for small $\varepsilon$

"That is, $\varphi$ satisfies the $\beta$-inequalities of Theorem 2.2.

${ }^{12}$ Since $f_{y}\left[u_{1}\right]=f_{y}\left[u_{2}\right] \equiv 0$ we examine $f_{y}\left[u_{1}, u_{2}\right]$ in order to determine stability; cf. H1 of $\$ 4$. 
to have a solution $y=y(t, \varepsilon)$ satisfying $y(t, \varepsilon) \rightarrow u_{1}(t),-1<t<t_{0}$, and $y(t, \varepsilon) \rightarrow u_{2}(t), t_{0} \leqslant t<1$, as $\varepsilon \rightarrow 0^{+}$, provided $u_{1}$ and $u_{2}$ are stable, i.e., satisfy $\mathrm{H} 1, \mathrm{H} 2$ or H3. A thorough discussion is given in [6].

EXAMPLE 6.4. We consider finally an application of Theorem 6.3. Take the problem

$$
\begin{aligned}
\varepsilon y^{\prime \prime} & =y-2 t y^{\prime}+y^{\prime 2}, \quad-1<t<1, \\
y(-1, \varepsilon) & =A, \quad y(1, \varepsilon)=B, \quad A>\frac{3}{4}, \frac{3}{4}<B<1 .
\end{aligned}
$$

The reduced solutions are $u_{L}(t)=\frac{3}{4} t^{2}$ and $u_{R}(t)$, which is given parametrically as $t=\frac{2}{3} \tau+c \tau^{-2}, u_{R}=2 t \tau-\tau^{2}, 0<\tau<(3 c)^{1 / 3}$, for some constant $c>0$, chosen so that $u_{R}(1)=B$. It happens that $u_{L}\left(t_{0}\right)=u_{R}\left(t_{0}\right)$, for a $t_{0}$ in $(0,1)$, and $u_{L}^{\prime}\left(t_{0}\right)=\frac{3}{2} t_{0}>u_{R}^{\prime}\left(t_{0}\right)=\frac{1}{2} t_{0}$. Since $f_{y}\left[u_{L}(t)\right]=t, f_{y^{\prime}}\left[u_{L}(t)\right]<0,-1$ $<t<-1+\delta$, and $f_{y}\left[u_{L}(t)\right]>0, t_{0}-\delta<t<t_{0}+\delta$, i.e., $u_{L}$ has the correct stability. On the other hand, $f_{y}\left[u_{R}(t)\right]=-\frac{2}{3} \tau+2 c \tau^{-2}<0, t_{0} \leqslant t<1$, and $f_{y} \equiv 1$. Next we observe that the crossing condition is satisfied:

$$
f\left(t_{0}, \frac{3}{4} t_{0}^{2}, \omega\right)=\left(\omega-\frac{3}{2} t_{0}\right)\left(\omega-\frac{1}{2} t_{0}\right)<0, \quad \frac{1}{2} t_{0}<\omega<\frac{3}{2} t_{0} .
$$

Finally $u_{L}(-1)<A$ and $f_{y^{\prime} y^{\prime}} \equiv 2$. Thus by Theorem 6.3 , the problem has a unique solution $y=y(t, \varepsilon)$ for which

$$
\begin{gathered}
\frac{3}{4} t^{2}-\varepsilon \gamma \leqslant y(t, \varepsilon)<\frac{3}{4} t^{2}+\left(A-\frac{3}{4}\right) \exp \left[-k(1+t) \varepsilon^{-1}\right]+\varepsilon \gamma, \quad-1<t<t_{0}, \\
u_{R}(t)-\varepsilon \gamma \leqslant y(t, \varepsilon) \leqslant u_{R}(t)+\varepsilon \gamma, \quad t_{0} \leqslant t \leqslant 1,
\end{gathered}
$$

where $0<k<1$.

7. Contrary $f_{y^{\prime}}$-stability. This section is devoted to a brief discussion of the case when the intersecting roots of the reduced equation have the "wrong" stability in a neighborhood of the point of intersection $t_{0}$. Recall that the reduced solution $u_{L}$ existing to the left of $t_{0}$ satisfied $f_{y}\left[u_{L}(t)\right]>0, t_{0}-\delta<t$ $\leqslant t_{0}$, while the reduced solution $u_{R}$ existing to the right of $t_{0}$ satisfied $f_{y}\left[u_{R}(t)\right]<0, t_{0} \leqslant t<t_{0}+\delta$. Such stability restrictions are the most natural ones and they have formed the basis for many treatments of problems of the form (3.1), (3.2). See, e.g., the discussions in [11], [12, Chapter 2], and [13]. However, it turns out that under special circumstances, the intersecting roots $u_{L}$ and $u_{R}$ can satisfy the opposite inequalities, i.e., $f_{y}\left[u_{L}(t)\right]<0$ and $f_{y}\left[u_{R}(t)\right]$ $\geqslant 0$, and yet, the solution of the full problem, for small $\varepsilon>0$, still treats the crossing as a Haber-Levinson one. The only requirements are that near $t=t_{0}$, the values of $f_{y^{\prime}}\left[u_{L}(t)\right]$ and $f_{y^{\prime}}\left[u_{R}(t)\right]$ are not too large and that the roots $u_{L}, u_{R}$ possess $f_{y}$-stability of the type discussed in $\S 4$. This type of result is illustrated in the following two theorems.

THEOREM 7.1. Assume 
(1) the reduced equation $f\left(t, u, u^{\prime}\right)=0$ has two solutions $u=u_{L}(t), u=$ $u_{R}(t)$, defined and of class $C^{(2)}$ on $\left[a, t_{0}\right],\left[t_{0}, b\right]$, respectively, and satisfying $u_{L}(a)=A, u_{L}\left(t_{0}\right)=u_{R}\left(t_{0}\right), u_{L}^{\prime}\left(t_{0}\right) \neq u_{R}^{\prime}\left(t_{0}\right)$, and $u_{R}(b)=B$;

(2) the function $f$ is continuous in all arguments and of class $C^{(1)}$ with respect to $y$ and $y^{\prime}$ in $Q: a \leqslant t \leqslant b, y-u(t)=O\left(\varepsilon^{1 / 2}\right), y^{\prime}-u^{\prime}(t)=O(d(t, \varepsilon))$, where

$$
u(t)= \begin{cases}u_{L}(t), & a \leqslant t \leqslant t_{0}, \\ u_{R}(t), & t_{0} \leqslant t \leqslant b,\end{cases}
$$

$d(t, \varepsilon)=O\left(\varepsilon^{1 / 2}\right)$, for $t$ in $[a, b] \backslash\left(t_{0}-\delta, t_{0}+\delta\right), d(t, \varepsilon)=O\left(\mid u_{L}^{\prime}\left(t_{0}\right)-\right.$ $\left.u_{R}^{\prime}\left(t_{0}\right) \mid\right)$, for $t$ in $\left(t_{0}-\delta, t_{0}+\delta\right)$; here $\delta>0$ is a small constant; in addition, $f=O\left(\left|y^{\prime}\right|^{2}\right)$, as $\left|y^{\prime}\right| \rightarrow \infty$;

(3) for $t$ in $\left(t_{0}-\delta, t_{0}\right], f_{y^{\prime}}\left(t, u_{L}(t), \xi_{1}\right) \leqslant 0, \xi_{1}-u_{L}^{\prime}(t)=O(d(t, \varepsilon))$, and for $t$ in $\left[t_{0}, t_{0}+\delta\right), f_{y^{\prime}}\left(t, u_{R}(t), \xi_{2}\right) \geqslant 0, \xi_{2}-u_{R}^{\prime}(t)=O(d(t, \varepsilon))$; in addition, either

$$
\begin{aligned}
& f_{y^{\prime}}\left(t, u_{L}(t), \xi_{1}\right)=O(\mu(\varepsilon)), \text { for } t \text { in }\left[t_{0}-\lambda(\varepsilon), t_{0}\right], \text { or } \\
& f_{y^{\prime}}\left(t, u_{R}(t), \xi_{2}\right)=O(\mu(\varepsilon)), \text { for } t \text { in }\left[t_{0}, t_{0}+\lambda(\varepsilon)\right],
\end{aligned}
$$

where $\mu(\varepsilon), \lambda(\varepsilon)$ are positive order functions such that $\mu(\varepsilon)=o(1), \lambda(\varepsilon)=o(1)$, and $\varepsilon^{1 / 2}=o(\lambda(\varepsilon))$

(4) there exists a positive constant $l$ such that

$$
f_{y}\left(t, u(t), u^{\prime}(t)\right) \geqslant l>0, \quad a \leqslant t \leqslant b .
$$

Then there exists an $\varepsilon_{0}>0$ such that for each $\varepsilon, 0<\varepsilon \leqslant \varepsilon_{0}$, the problem (3.1), (3.2) has a solution $y=y(t, \varepsilon)$. Moreover, for

$$
\begin{aligned}
u(t) & = \begin{cases}u_{L}(t), & a \leqslant t \leqslant t_{0}, \\
u_{R}(t), & t_{0} \leqslant t \leqslant b,\end{cases} \\
y(t, \varepsilon) & =u(t)+O\left(\max \left\{\varepsilon^{1 / 2}, \mu(\varepsilon)\right\}\right), \quad a \leqslant t \leqslant b .
\end{aligned}
$$

PROof. The theorem is proved by applying Theorem 2.2. Suppose, for example, that $u_{L}^{\prime}\left(t_{0}\right)<u_{R}^{\prime}\left(t_{0}\right)$, and that $f_{y^{\prime}}\left(t, u_{R}(t), \xi_{2}\right)=O(\mu(\varepsilon))$, for $t$ in $\left[t_{0}, t_{0}+\lambda(\varepsilon)\right]$, then we define for $\varepsilon>0$

$$
\begin{gathered}
\alpha(t, \varepsilon)=\left\{\begin{aligned}
u_{L}(t)-\varepsilon \gamma l^{-1}, & a \leqslant t \leqslant t_{0}, \\
u_{R}(t)-\varepsilon \gamma l^{-1}, & t_{0} \leqslant t \leqslant b,
\end{aligned}\right. \\
\beta(t, \varepsilon)=\left\{\begin{array}{r}
u_{L}(t)+\nu(\varepsilon) \gamma_{1} l^{-1}, a \leqslant t \leqslant t_{0}, \\
u_{R}(t)+\left(\varepsilon l^{-1}\right)^{1 / 2}\left(u_{R}^{\prime}\left(t_{0}\right)-u_{L}^{\prime}\left(t_{0}\right)\right) \exp \left[\begin{array}{r}
\left.-\left(\varepsilon^{-1} l\right)^{1 / 2}\left(t-t_{0}\right)\right] \\
+\nu(\varepsilon) \gamma_{2} l^{-1}, \quad t_{0} \leqslant t \leqslant b,
\end{array}\right.
\end{array}\right.
\end{gathered}
$$

where $\nu(\varepsilon)=\max \left\{\varepsilon^{1 / 2}, \mu(\varepsilon)\right\}$ and the $\gamma_{1}, \gamma_{2}$ are chosen sufficiently large and to make $\beta(t, \varepsilon)$ well defined. It is now straightforward to verify that $\alpha$ and $\beta$ satisfy the inequalities of Theorem 2.2. 
We discuss now the boundary layer analog of Theorem 7.1, that is, we give sufficient conditions for the existence of a solution possessing boundary layer behavior at one or both endpoints.

\section{THEOREM 7.2. Assume}

(1) the reduced equation $f\left(t, u, u^{\prime}\right)=0$ has two solutions $u=u_{L}(t), u=$ $u_{R}(t)$, defined and of class $C^{(2)}$ on $\left[a, t_{0}\right],\left[t_{0}, b\right]$, respectively, with $u_{L}\left(t_{0}\right)=$ $u_{R}\left(t_{0}\right)$ and $u_{L}^{\prime}\left(t_{0}\right) \neq u_{R}^{\prime}\left(t_{0}\right)$;

(2) the function $f$ is continuous in all arguments and of class $C^{(2)}$ with respect to $y$ and $y^{\prime}$ in $\mathcal{P}: a \leqslant t \leqslant b, y-u(t)=O(d(t, \varepsilon)), y^{\prime}-u^{\prime}(t)=O\left(d_{1}(t, \varepsilon)\right)$, where

$$
\begin{gathered}
u(t)= \begin{cases}u_{L}(t), & a \leqslant t \leqslant t_{0}, \\
u_{R}(t), & t_{0} \leqslant t \leqslant b,\end{cases} \\
d(t, \varepsilon)=O\left(\left|A-u_{L}(a)\right|\right)+O\left(\varepsilon^{1 / 2}\right), \quad a \leqslant t<a+\delta, \\
d(t, \varepsilon)=O\left(\left|B-u_{R}(b)\right|\right)+O\left(\varepsilon^{1 / 2}\right), \quad b-\delta<t \leqslant b,
\end{gathered}
$$

and $d(t, \varepsilon)=O\left(\varepsilon^{1 / 2}\right), a+\delta \leqslant t \leqslant b-\delta ; d_{1}(t, \varepsilon)=O\left(\varepsilon^{-1}\right), a \leqslant t<a+$ $\delta$, if $u_{L}(a) \neq A, d_{1}(t, \varepsilon)=O\left(\varepsilon^{-1}\right), b-\delta<t \leqslant b$, if $u_{R}(b) \neq B, d_{1}(t, \varepsilon)=$ $O\left(\left|u_{L}^{\prime}\left(t_{0}\right)-u_{R}^{\prime}\left(t_{0}\right)\right|\right), t_{0}-\delta<t<t_{0}+\delta, d_{1}(t, \varepsilon)=O\left(\varepsilon^{1 / 2}\right),\{a+\delta \leqslant t \leqslant$ $\left.t_{0}-\delta\right\} \cup\left\{t_{0}+\delta \leqslant t \leqslant b-\delta\right\} ;$ moreover, $f_{y^{\prime} y^{\prime}}=O(1)$, as $\left|y^{\prime}\right| \rightarrow \infty$;

(3) for $t$ in $\left(t_{0}-\delta, t_{0}\right], f_{y^{\prime}}\left(t, u_{L}(t), \xi_{1}\right) \leqslant 0, \xi_{1}-u_{L}^{\prime}(t)=O\left(d_{1}(t, \varepsilon)\right)$, and if $u_{L}(a) \neq A, f_{y^{\prime}}\left(t, u_{L}(t), u_{L}^{\prime}(t)\right) \leqslant-k<0, a \leqslant t<a+\delta$ for some positive constant $k$; similarly, for $t$ in $\left[t_{0}, t_{0}+\delta\right), f_{y^{\prime}}\left(t, u_{R}(t), \xi_{2}\right) \geqslant 0, \xi_{2}-u_{R}^{\prime}(t)=$ $O\left(d_{1}(t, \varepsilon)\right)$, and if $u_{R}(b) \neq B, f_{y^{\prime}}\left(t, u_{R}(t), u_{R}^{\prime}(t)\right) \geqslant k>0$, in addition, either $f_{y^{\prime}}\left(t, u_{L}(t), \xi_{1}\right)=O(\mu(\varepsilon))$, for $t$ in $\left(t_{0}-\lambda(\varepsilon), t_{0}\right]$ or $f_{y^{\prime}}\left(t, u_{R}(t), \xi_{2}\right)=O(\mu(\varepsilon))$, for $t$ in $\left[t_{0}, t_{0}+\lambda(\varepsilon)\right)$, where $\mu(\varepsilon), \lambda(\varepsilon)$ are positive order functions such that $\mu(\varepsilon)=o(1), \lambda(\varepsilon)=o(1)$ and $\varepsilon^{1 / 2}=o(\lambda(\varepsilon))$;

(4) there exists a positive constant $l$ such that $f_{y}\left(t, u(t), u^{\prime}(t)\right) \geqslant l>0$, $a+\delta \leqslant t \leqslant b-\delta$;

(5) there exists a positive constant $p$ such that $\left(A-u_{L}(a)\right) f_{y^{\prime} y^{\prime}} \geqslant p>0$, in $\mathcal{P} \cap[a, a+\delta)$, if $u_{L}(a) \neq A ;\left(B-u_{R}(b)\right) f_{y^{\prime} y^{\prime}} \geqslant p>0$, in $\mathcal{P}^{\prime} \cap(b-\delta, b]$, if $u_{R}(b) \neq B$.

Then there exists an $\varepsilon_{0}>0$ such that for each $\varepsilon, 0<\varepsilon \leqslant \varepsilon_{0}$, there exists a solution $y=y(t, \varepsilon)$ of (3.1), (3.2) such that, if

$$
\begin{aligned}
u(t)= \begin{cases}u_{L}(t), & a \leqslant t \leqslant t_{0}, \\
u_{R}(t), & t_{0} \leqslant t \leqslant b,\end{cases} \\
y(t, \varepsilon)=u(t)+O\left(\left|A-u_{L}(a)\right| \exp \left[-\bar{k}(1+t) \varepsilon^{-1}\right]\right) \\
+O\left(\left|B-u_{R}(b)\right| \exp \left[-\bar{k}(1-t) \varepsilon^{-1}\right]\right) \\
+O\left(\max \left\{\varepsilon^{1 / 2}, \mu(\varepsilon)\right\}\right), \quad a \leqslant t \leqslant b, \quad 0<\bar{k}<k .
\end{aligned}
$$


This theorem is proved in the same manner as Theorem 7.1 by adding the appropriate boundary layer terms to the functions $\alpha$ and $\beta$ given above. The result can be viewed as a generalization of Theorem 4 in [2]. We further remark that these theorems can be extended in an obvious way to the case of more general $f_{y}$-stability as discussed above.

Some applications of these theorems are now given.

EXAMPLE 7.1. As an example of a case of one-sided contrary $f_{y^{\prime}}$-stability, consider

$$
\begin{gathered}
\varepsilon y^{\prime \prime}=t y^{\prime 2}+t y^{\prime}+y, \quad-1<t<1, \\
y(-1, \varepsilon)=2, \quad y(1, \varepsilon)=0 .
\end{gathered}
$$

The reduced solutions of interest are $u_{L}(t)=-2 t$ and $u_{R}(t) \equiv 0$, which have an angular crossing at $t_{0}=0$. As regards $u_{L}, f_{y}\left[u_{L}(t)\right]=-3 t>0,-1 \leqslant t \leqslant$ 0 , i.e., $u_{L}$ is stable on $[-1,0)$; however, $f_{y}\left[u_{R}(t)\right]=t \geqslant 0,0 \leqslant t \leqslant 1$, so that $u_{R}$ has the wrong stability on $[0,1]$. In particular, $f_{y^{\prime}}\left(t, u_{R}(t), \xi\right)=t(2 \xi+1)=$ $O\left(\varepsilon^{1-\rho}\right), 0 \leqslant t \leqslant \lambda(\varepsilon)=\varepsilon^{1-\rho}, \frac{1}{2}<\rho<1$, and $f_{y} \equiv 1$, so we can apply Theorem 7.1 to conclude that this problem has a unique solution $y=y(t, \varepsilon)$ for which

$$
y(t, \varepsilon)=\max \{-2 t, 0\}+O\left(\varepsilon^{1-\rho}\right), \quad-1 \leqslant t \leqslant 1 .
$$

We note that if the above problem is furnished with the boundary conditions $y(-1, \varepsilon)=2, y(1, \varepsilon)=B>0$, then for small $\varepsilon>0$, the solution passesses, in addition to Haber-Levinson behavior at $t_{0}=0$, boundary layer behavior at $t=1$. This follows directly from Theorem 7.2 since $f_{y}\left[u_{R}(t)\right]>0$, for $t$ in $(1-\delta, 1], u_{R}(1)<B$, and $f_{y^{\prime} y^{\prime}} \equiv 2 t>0$, for $t$ in $(1-\delta, 1]$. Indeed we have the estimate

$$
\begin{aligned}
\max \{-2 t, 0\}-\varepsilon^{1-\rho} \gamma \leqslant y(t, \varepsilon)< & \max \{-2 t, 0\}+B \exp \left[-k(1-t) \varepsilon^{-1}\right] \\
& +\varepsilon^{1-\rho} \gamma,-1 \leqslant t \leqslant 1,
\end{aligned}
$$

where $0<k<1$.

EXAMPLE 7.2. We consider next an example with a slightly more subtle form of contrary stability, namely,

$$
\begin{aligned}
\varepsilon y^{\prime \prime} & =t y^{\prime 2}-y y^{\prime}, \quad-1<t<1, \\
y(-1, \varepsilon) & =A, \quad y(1, \varepsilon)=B, \quad A>0, B<0, A>-2 B .
\end{aligned}
$$

The reduced pair to consider is $u_{L}(t)=-A t$ and $u_{R}(t)=B t$. We observe that $f_{y}\left[u_{L}(t)\right]=-A t>0,-1 \leqslant t<0$ and $f_{y}\left[u_{R}(t)\right]=B t<0,0<t<1$; however,

$$
f_{y^{\prime}}\left(t, u_{L}(t), \xi\right)=(2 \xi+A) t \ngtr 0,
$$

$-\delta<\mathrm{t}<0,-A<\xi<B$. Consequently we cannot apply Theorem 5.1 
since the domain of stability of $u_{L}$ is not large enough. Nevertheless, $f_{y^{\prime}}\left(t, u_{L}(t), \xi\right)=O(t), f_{y^{\prime}}\left(t, u_{R}(t), \xi\right)=O(t), f_{y}\left[u_{L}(t)\right] \equiv A>0$, and $f_{y}\left[u_{R}(t)\right]$ $\equiv-B>0$, so we can apply Theorem 7.1 to deduce the existence of a solution $y=y(t, \varepsilon)$ such that

$$
\begin{aligned}
\max \{-A t, B t\} \leqslant y(t, \varepsilon) \leqslant \max \{-A t, B t\} & +\varepsilon^{1-\rho} \gamma, \\
-1 & \leqslant t \leqslant 1, \frac{1}{2}<\rho<1 .
\end{aligned}
$$

EXAMPLE 7.3. We give finally an example of a Haber-Levinson crossing with two-sided contrary $f_{y^{\prime}}$-stability. Take the problem

$$
\begin{gathered}
\varepsilon y^{\prime \prime}= \begin{cases}y+t y^{\prime}-t y^{\prime 2}+3 t, & -1 \leqslant t \leqslant 0, \\
y+t y^{\prime}+t y^{\prime 2}, & 0 \leqslant t \leqslant 1,\end{cases} \\
y(-1, \varepsilon)=A \geqslant 1, \quad y(1, \varepsilon)=B \geqslant 0,
\end{gathered}
$$

and consider the reduced solutions $u_{L}(t)=-t$ and $u_{R}(t) \equiv 0$. Here $t_{0}=0$, $f_{y}\left[u_{L}(t)\right]=3 t<0,-1 \leqslant t \leqslant 0$, and $f_{y}\left[u_{R}(t)\right]=t \geqslant 0,0 \leqslant t \leqslant 1$. If $A=1$ and $B=0$, we can apply Theorem 7.1 to deduce the existence of a unique solution $y=y(t, \varepsilon)$ satisfying

$$
y(t, \varepsilon)=\max \{-t, 0\}+O\left(\varepsilon^{1-\rho}\right), \quad-1 \leqslant t \leqslant 1, \frac{1}{2}<\rho<1 .
$$

More generally, if $A \geqslant 1$ and $B \geqslant 0$, since $u_{L}$ and $u_{R}$ are stable with respect to boundary layers at $t=-1$ and $t=1$, respectively, and since $f_{y^{\prime} y^{\prime}}=2|t|>0,-1 \leqslant t \leqslant-1+\delta$ and $1-\delta \leqslant t \leqslant 1$, we apply Theorem 7.2 to deduce the existence of a unique solution $y=y(t, \varepsilon)$ for which

$$
\begin{aligned}
\max \{-t, 0\}-\varepsilon^{1-\rho} \gamma & \\
\leqslant & y(t, \varepsilon) \leqslant \max \{-t, 0\}+(A-1) \exp \left[-k_{1}(1+t) \varepsilon^{-1}\right] \\
& +B \exp \left[-k_{2}(1-t) \varepsilon^{-1}\right]+\varepsilon^{1-\rho} \gamma, \quad-1<t \leqslant 1,
\end{aligned}
$$

where $0<k_{1}<3,0<k_{2}<1$ and $\gamma$ is a positive constant.

8. Concluding remarks. In the sections above we have indicated how solutions of certain boundary value problems can behave roughly like those first studied by Haber and Levinson when their original stability assumptions are modified. Our approach has been based largely on the observation that it is the interplay between $y^{\prime}$-stability and $y$-stability which ultimately determines the behavior of solutions for small $\varepsilon>0$. In general, to study the existence and the asymptotic behavior of solutions in cases where $f_{y^{\prime}}$ is zero at isolated points or along various curves one must check the reduced solutions for $y$-stability before reaching conclusions based solely on the presence (or absence) of $y^{\prime}$-stability. This is particularly important when some reduced solutions possess the weak instability described in $\$ 7$. In this regard a priori estimates which establish bounds on solutions may be of use in obtaining 
more precise information on those solutions which have boundary and interior layer behavior.

ACKNOWLEDGEMENT. The author gratefully acknowledges the support provided him by the Mathematics Department of the University of Wisconsin at Madison. He also wishes to thank Professor Wolfgang R. Wasow for his hospitality during the visit to Wisconsin.

\section{REFERENCES}

1. Yu. P. Boglaev, The two-point problem for a class of ordinary differential equations with a small parameter coefficient of the derivative, USSR Computational Math. and Math. Phys. 10 (1970), 191-204.

2. N. I. Briš, On boundary value problems for the equation $\varepsilon y^{\prime \prime}=f\left(x, y, y^{\prime}\right)$ for small $\varepsilon$, Dokl. Akad. Nauk SSSR 95 (1954), 429-432.

3. F. W. Dorr, S. V. Parter and L. F. Shampine, Applications of the maximum principle to singular perturbation problems, SIAM Review 15 (1973), 43-88.

4. S. Haber and N. Levinson, $A$ boundary value problem for a singularly perturbed differential equation, Proc. Amer. Math. Soc. 6 (1955), 866-872.

5. P. Habets and M. Laloy, Etude de problemes aux limites par la methode des sur-et sous solutions, Lecture Notes, Catholic Univ. of Louvain, 1974.

6. F. A. Howes, $A$ class of boundary value problems whose solutions possess angular limiting behavior, Rocky Mountain J. Math., 6 (1976), 591-607.

7. Singularly perturbed nonlinear boundary value problems with turning points. II, SIAM J. Math. Anal. (to appear).

8. M. Nagumo, Uber die Differentialgleichung $y^{\prime \prime}=f\left(x, y, y^{\prime}\right)$, Proc. Phys. Math. Soc. Japan 19 (1937), 861-866.

9. R. E. O'Malley, Jr., On singular perturbation problems with interior nonuniformities, J. Math. Mech. 19 (1970), 1103-1112.

10. K. Schmitt, Boundary value problems for nonlinear second order differential equations, Monaish. Math. 72 (1968), 347-354.

11. A. B. Vasil'eva, Uniform approximation to the solution of a set of simultaneous differential equations involving a small parameter in the derivative and its application to boundary value problems, Dokl. Akad. Nauk SSSR 124 (1959), 509-512.

12. Asymptotic behavior of solutions to certain problems involving nonlinear differential equations containing a small parameter multiplying the highest derivatives, Russian Math. Surveys 18 (1963), 13-84.

13. , Asymptotic methods in the theory of ordinary differential equations containing small parameters in front of the higher derivatives, USSR Computational Math. and Math. Phys. 3 (1963), 823-863.

14. M. I. Vishik and L. A. Lyusternik, Initial jump for nonlinear differential equations containing a small parameter, Soviet Math. Dokl. 1 (1960), 749-752.

15. W. R. Wasow, Asymptotic expansions for ordinary differential equations, "MIR," Moscow, 1968. (Russian edition of the book originally published by Wiley, New York, 1965.)

Department of Mathematics, University of Wisconsin, Madison, Wisconsin 53706

Current address: School of Mathematics, University of Minnesota, Minneapolis, Minnesota 55455 